\title{
Topological structure of a Nambu monopole in two-Higgs-doublet models: Fiber bundle, Dirac's quantization, and a dyon
}

\author{
Minoru Eto, ${ }^{1,2}$ Yu Hamada $\odot{ }^{3}$ and Muneto $\mathrm{Nitta}^{4,2}$ \\ ${ }^{1}$ Department of Physics, Yamagata University, Kojirakawa-machi 1-4-12, \\ Yamagata, Yamagata 990-8560, Japan \\ ${ }^{2}$ Research and Education Center for Natural Sciences, Keio University, \\ 4-1-1 Hiyoshi, Yokohama, Kanagawa 223-8521, Japan \\ ${ }^{3}$ Department of Physics, Kyoto University, Kitashirakawa, Kyoto 606-8502, Japan \\ ${ }^{4}$ Department of Physics, Keio University, 4-1-1 Hiyoshi, Kanagawa 223-8521, Japan
}

(Received 8 August 2020; accepted 20 October 2020; published 23 November 2020)

\begin{abstract}
We find a topologically nontrivial structure of the Nambu monopole in the two-Higgs-doublet model (2HDM), which is a magnetic monopole attached by two topologically stable $Z$ strings ( $Z$ flux tubes) from two opposite sides. The structure is in sharp contrast to the topological triviality of the Nambu monopole in the Standard Model (SM), which is attached by a single nontopological $Z$ string. It is found that the Nambu monopole in the 2HDM possesses the same fiber-bundle structure as those of the 't Hooft-Polyakov monopole and the Wu-Yang description of the Dirac monopole, as a result of the fact that the electromagnetic gauge field is well defined even inside the strings and is nontrivially fibered around the monopole, while the Nambu monopole in the SM is topologically trivial because electroweak gauge symmetry is restored at the core of the string. Consequently, the Nambu monopole in the 2HDM can be regarded as an embedding of the 't Hooft-Polyakov monopole into the $S U(2)_{W}$ gauge symmetry, and the Dirac quantization condition always holds, which is absent for the Nambu monopole in the SM. Furthermore, we construct a dyon configuration attached with the two strings.
\end{abstract}

DOI: 10.1103/PhysRevD.102.105018

\section{INTRODUCTION}

Magnetic monopoles have attracted great interest from many physicists since the seminal work by Dirac [1], which improved the asymmetry between electric and magnetic charges in the Maxwell equations and provided an explanation for the electric charge quantization. While the monopole originally suggested by Dirac (Dirac monopole) is a singular point-like object accompanied by an infinitely thin unphysical solenoid string (Dirac string), it was reformulated by $\mathrm{Wu}$ and Yang [2] from the viewpoint of fiber bundles without any string singularities.

In field theories, magnetic monopoles often appear as topological solitons resulting from a nontrivial second homotopy group $\pi_{2}$ of the vacuum manifold, which were first discovered by 't Hooft and Polyakov $[3,4]$ in the $S O(3)$ Georgi-Glashow model [5]. In such a model, the electric charge quantization can be understood by the compactness of the unbroken $U(1)$ subgroup. In the same model, Julia

Published by the American Physical Society under the terms of the Creative Commons Attribution 4.0 International license. Further distribution of this work must maintain attribution to the author(s) and the published article's title, journal citation, and DOI. Funded by SCOAP ${ }^{3}$. and Zee [6] discovered a solution with both the electric and magnetic charges, called a dyon. While the magnetic monopoles and dyons theoretically play crucial roles in understanding nonperturbative aspects of (non)supersymmetric field theories [7-9], experimentally such monopoles have never been found in reality, except for condensedmatter analogues $[10,11]$.

A magnetic monopole configuration in the Standard Model (SM) was first considered by Nambu [12], which is called the Nambu monopole. Since the Nambu monopole is attached by a vortex string, which is a physical string with finite thickness (unlike the Dirac string for the Dirac monopole), it is pulled by the tension of the string and cannot be stable. Nevertheless, the Nambu monopole may be phenomenologically and cosmologically useful; for instance, it has been suggested that it produces primordial magnetic fields before their disappearance $[13,14]$. The electric charge quantization and the dyon associated with the Nambu monopole were also considered in Ref. [15]. The mathematical reason for the instability is its trivial topology, that is, the vacuum manifold of the SM is $S^{3}$ with a trivial second homotopy group $\pi_{2}$. Likewise, the vacuum manifold $S^{3}$ has a trivial $\pi_{0}$ for domain walls and a trivial $\pi_{1}$ for cosmic strings. Nontopological electroweak $Z$ strings (or magnetic $Z$ fluxes) [16-21] have been studied 
extensively, but they were shown to be unstable for the observed values of the Higgs mass $m_{h} \simeq 125 \mathrm{GeV}$ and the Weinberg angle $\sin ^{2} \theta_{W} \simeq 0.23$ [22,23]. The Nambu monopoles are the end points of these electroweak $Z$ strings [12].

On the other hand, the two-Higgs-doublet model (2HDM) - in which one more Higgs doublets is added to the SM-is one of the most popular extensions of the SM with the potential to resolve many unsolved problems of the SM (for reviews, see, e.g., Refs. [24,25]). Two-Higgsdoublet fields also appear in supersymmetric extensions of the SM [26,27]. In addition to the $125 \mathrm{GeV}$ Higgs boson it has four additional Higgs bosons, which could be directly produced at the LHC, though there has been no signal thus far, therefore placing lower bounds on the masses of these additional scalar bosons. These lower bounds highly depend on the parameter choices of the 2HDM. For more detailed phenomenological studies, see, e.g., Refs. [28-32] and references therein. One of the most remarkable aspects of the 2HDM that distinguishes it from the SM may be that it has a much richer vacuum structure than the SM, thereby allowing for a variety of topologically stable solitons, in addition to nontopological solitons [33-41] analogous to the SM: domain walls [42-47], membranes [48,49], and cosmic strings such as topological $Z$ strings $[44,45,50,51]$ (see also Ref. [52]). In particular, it was found $[44,45]$ that the topological $Z$ strings in Refs. [50,51] are global strings confining non-Abelian fluxes in the cores, and are accompanied by non-Abelian moduli, analogous to non-Abelian strings in dense QCD [53-57].

In our previous papers $[58,59]$ we studied the Nambu monopole in the $2 \mathrm{HDM}$, which is a magnetic monopole attached with two topological $Z$ strings from two opposite sides. The monopole is a regular solution of the equation of motion (EoM) and is topologically and dynamically stable when the Higgs potential has two global symmetries: a global $U(1)$ symmetry that ensures the stability of the topological $Z$ strings, and a discrete symmetry $\mathbb{Z}_{2}$ exchanging the topological $Z$ strings. The string tensions pulling the monopole are balanced due to the $\mathbb{Z}_{2}$ symmetry and the monopole does not move (unlike the Nambu monopole in the SM), and it can be regarded as a topologically stable $\mathbb{Z}_{2}$ kink on one string. Once the $\mathbb{Z}_{2}$ symmetry is explicitly broken it starts to move along the string [59], and if the global $U(1)$ symmetry is broken the string is attached by a domain wall $[44,45,50,51]$.

One might wonder if the Nambu monopole in the 2HDM is not a true magnetic monopole from several viewpoints. First, its topological features look different from those of the ordinary magnetic monopole, such as the 't HooftPolyakov monopole, because the monopole is not isolated but rather is attached with the two $Z$ string. In other words, there is no nontrivial $\pi_{2}$ as in the SM. Second, the magnetic charge of the monopole, $4 \pi \sin \theta_{W} / e$, is not an integer multiple of $2 \pi / e$ (where $e$ is the electromagnetic coupling constant), and it seems inconsistent with the Dirac quantization condition.

In this paper we resolve the above two mysteries. We show that the Nambu monopole in the 2HDM has the same topological structure as the 't Hooft-Polyakov monopole in a certain sense, while the Nambu monopole in the SM is topologically trivial. We consider an infinitely large sphere $S^{2}$ surrounding the monopole which the two $Z$ strings pass through, and investigate the fiber bundle of the electromagnetic gauge field on the base space $S^{2}$. We find that the electromagnetic field is well defined and regular everywhere on the sphere $S^{2}$ and is nontrivially fibered like the Wu-Yang description of the Dirac monopole, despite the trivial $\pi_{2}$. ${ }^{1}$ This is a remarkable difference from the Nambu monopole in the SM, in which the electromagnetic gauge field cannot be defined at the center of the nontopological $Z$ string attached to the monopole since the electroweak gauge symmetry is restored there. It has a topologically trivial fiber bundle structure unlike the Wu-Yang fiber bundle. ${ }^{2}$ Interestingly, the Nambu monopole in the 2HDM can be regarded as an embedding of the 't Hooft-Polyakov monopole into the $S U(2)_{W}$ sector. Furthermore, we derive the Dirac quantization condition from the single valuedness of wave functions around the nontrivially fibered electromagnetic field. We find that the condition always holds if the $Z$ flux is taken into account as well, and thus there is no inconsistency. This can be also understood from the fact that the presence of the $Z$ strings cannot be ignored because they are observable even at the classical level, unlike the Dirac string which is unobservable. We also consider a dyon configuration in the $2 \mathrm{HDM}$ by describing a timedependent ansatz and give a general quantization condition for the dyon charges, as in Ref. [15].

This paper is organized as follows. In Sec. II we give a brief review of the 't Hooft-Polyakov monopole and consider the fiber bundle and the Dirac quantization condition. In Sec. III we revisit the Nambu monopole in the SM and contrast its topological properties with the 't Hooft-Polyakov case. In Sec. IV, for later use, we give a general definition for the electromagnetic gauge field and the field strengths in the 2HDM. Then we consider the topological properties of the electromagnetic gauge in Sec. V. The dyon configuration in the $2 \mathrm{HDM}$ is considered in Sec. VI. Our conclusion and discussion are presented in Sec. VII. In Appendix A we present some useful relations.

\footnotetext{
${ }^{1}$ Note that a nontrivial $\pi_{2}$ is sufficient for a nontrivial fiber of the electromagnetic gauge field because of the mathematical relation $\pi_{2}(G / H)=\pi_{1}(H)$, where $G$ is a simple gauge group breaking into $H$. Nevertheless, it is not necessary.

${ }^{2}$ The author of Ref. [15] insisted that the magnetic charge of the Nambu monopole in the SM is topologically protected, but we show that this is not the case. The author of Ref. [15] probably did not pay enough attention to the fact that the electromagnetic gauge symmetry is not defined at the center of the $Z$ string due to the vanishing field value of the Higgs doublet.
} 
In Appendix B we consider the background gauge condition (which is used in Sec. VI). In Appendix C we present a description in the singular gauge for the Nambu monopole in the 2HDM.

\section{REVIEW OF THE 'T HOOFT-POLYAKOV MONOPOLE}

\section{A. The 't Hooft-Polyakov monopole}

In this section we briefly review the 't Hooft-Polyakov monopole, with a particular emphasis on its topological properties. For more details, see, e.g., Ref. [60]. We start with the Yang-Mills-Higgs model with the $S U(2)$ gauge group:

$$
\mathcal{L}=\frac{-1}{4} W_{\mu \nu}^{a} W^{\mu \nu a}+\left(D_{\mu} \Phi\right)^{a}\left(D^{\mu} \Phi\right)^{a}-V(\Phi),
$$

with the potential term

$$
V(\Phi)=-m_{\Phi}^{2}\left(\Phi^{a}\right)^{2}+\lambda\left(\left(\Phi^{a}\right)^{2}\right)^{2},
$$

where $\Phi^{a}(a=1,2,3)$ is the Higgs field in the adjoint representation ${ }^{3}$ and $W_{\mu}^{a}$ is the gauge field with field strength $W_{\mu \nu}^{a} \equiv \partial_{\mu} W_{\nu}^{a}-\partial_{\nu} W_{\mu}^{a}-g \epsilon^{a b c} W_{\mu}^{b} W_{\nu}^{c}$. The Higgs vacuum is given by

$$
\Phi_{0}^{a}=(0,0, v)^{t},
$$

with $v \equiv m_{\Phi} / \sqrt{2 \lambda}$. The vacuum (2.3) spontaneously breaks $S U(2)$ into $U(1)$.

Let us consider a 't Hooft-Polyakov monopole located at the origin. In this paper, it is enough to consider an asymptotic configuration describing the monopole at large distances $r \rightarrow \infty$, given by

$\left.\Phi^{a}(\theta, \varphi)\right|_{\infty}=v \frac{x^{a}}{r},\left.\quad g W_{i}^{a}(\theta, \varphi)\right|_{\infty}=\epsilon^{i a b} \frac{x^{b}}{r^{2}}$,

where $\theta$ and $\varphi$ are the zenith and azimuth angles, respectively. This is sometimes called the hedgehog gauge. In this ansatz, an unbroken gauge group is determined by a unit vector $n^{a}$ defined by

$$
n^{a} \equiv \frac{\Phi^{a}}{|\Phi|}
$$

with $|\Phi| \equiv \sqrt{\Phi^{a} \Phi^{a}}$. The vector $n^{a}$ is regular everywhere except for a region in which $|\Phi|=0$ (corresponding to the center of the monopole). We denote the unbroken gauge group by $U(1)_{\mathrm{EM}}$. Indeed, the Higgs field in Eq. (2.4) is invariant under the $U(1)_{\mathrm{EM}}$ rotation:

\footnotetext{
${ }^{3}$ Note that the true gauge group is $S U(2) / \mathbb{Z}_{2}$ in the presence of the adjoint representation only. Nevertheless, for ease of notation we will use $S U(2)$, which is the universal covering of $S O(3)$.
}

$$
e^{-i \alpha n^{a} \sigma^{a}} \Phi e^{i \alpha n^{b} \sigma^{b}}=\Phi,
$$

with $\Phi=\Phi^{a} \sigma^{a} / 2$, and $\alpha$ is an arbitrary constant. From Eq. (2.4), $n^{a}$ has a hedgehog structure at large distances, $n^{a} \sim x^{a} / r$, and hence is a map from the two-dimensional sphere $S^{2}$ spanned by $(\theta, \varphi)$ at spatial infinity onto a twodimensional internal sphere $S^{2} \simeq\left\{n^{a} \mid\left(n^{a}\right)^{2}=1\right\}$ with a unit winding number:

$$
\frac{1}{8 \pi} \int_{S^{2}} d S_{k} \epsilon^{i j k} \epsilon^{a b c} n^{a} \partial_{i} n^{b} \partial_{j} n^{c}=1,
$$

where the integration is performed on the two-dimensional sphere surrounding the monopole at the spatial infinity. In addition, at large distances it satisfies the following identity:

$$
\left(D_{\mu} n\right)^{a}=\partial_{\mu} n^{a}-g \epsilon^{a b c} W_{\mu}^{b} n^{c}=0 .
$$

The electromagnetic field strength is defined by a projection of the $S U(2)$ field strength onto the $U(1)_{\mathrm{EM}}$ subgroup,

$$
\begin{aligned}
F_{i j}^{\mathrm{EM}} & \equiv W_{i j}^{a} n^{a} \\
& \sim \frac{1}{g} \epsilon_{i j k} \frac{x^{k}}{r^{3}} \quad(\text { for } r \rightarrow \infty),
\end{aligned}
$$

and the magnetic field is

$$
B_{i} \equiv \frac{1}{2} \epsilon_{i j k} F_{j k}^{\mathrm{EM}} \sim \frac{1}{g} \frac{x^{i}}{r^{3}} .
$$

Therefore, the ansatz (2.4) describes a magnetic monopole with a magnetic charge $q_{M}=\int_{S^{2}} d S_{i} B_{i}=4 \pi / g$. Note that, if one defines the electromagnetic gauge field $A_{\mu}$ as

$$
A_{\mu} \equiv W_{\mu}^{a} n^{a},
$$

then Eq. (2.9) can be rewritten as

$$
F_{i j}^{\mathrm{EM}}=\partial_{i} A_{j}-\partial_{j} A_{i}+\frac{1}{g} \epsilon^{a b c} n^{a} \partial_{i} n^{b} \partial_{j} n^{c},
$$

where we have used Eq. (2.8). Thus, the electromagnetic field strength is composed of a contribution purely from the electromagnetic gauge field $A_{\mu}$ and an additional contribution from the Higgs field $\Phi$, which explains why there can be a nonzero divergence of the magnetic flux.

We can consider a more general case where the magnetic charge is larger than $4 \pi / g$. This is achieved by considering an ansatz for $\Phi^{a}$ or $n^{a}$ with a general winding number, i.e.,

$$
\frac{1}{8 \pi} \int_{S^{2}} d S_{k} \epsilon^{i j k} \epsilon^{a b c} n^{a} \partial_{i} n^{b} \partial_{j} n^{c}=m,
$$


where $m$ must be an integer since it is a topological number. In other words, such a configuration is in a topological sector labeled by $m \in \mathbb{Z}$ of the second homotopy group $\pi_{2}[S U(2) / U(1)] \simeq \pi_{2}\left(S^{2}\right)=\mathbb{Z}$. The magnetic charge is obtained by integrating Eq. (2.13) on $S^{2}$,

$$
q_{M}=\int_{S^{2}} d S_{k} \epsilon^{i j k} F_{i j}^{\mathrm{EM}}=\frac{4 \pi}{g} m
$$

where we have used the fact that the integration for $A_{i}$ vanishes as long as $A_{i}$ is regular. Thus, $q_{M}$ is quantized with the topological number $m$.

\section{B. Singular gauge for the 't Hooft-Polyakov monopole}

It is instructive to see the 't Hooft-Polyakov monopole in a singular gauge (also called the string gauge). First, let us consider the single monopole configuration with $m=1$ in Eq. (2.4). To move from the hedgehog gauge to the string gauge, we introduce a gauge transformation $U$ such that

$$
n^{a} \frac{\sigma^{a}}{2} \rightarrow\left(n^{\prime}\right)^{a} \frac{\sigma^{a}}{2}=U^{\dagger} n^{a} \frac{\sigma^{a}}{2} U=\frac{\sigma^{3}}{2}
$$

or, equivalently, $\quad n^{a}=(\sin \theta \cos \varphi, \sin \theta \sin \varphi, \cos \theta)^{t} \rightarrow$ $\left(n^{\prime}\right)^{a}=(0,0,1)^{t}$. Such a transformation $U$ is given by

$$
\begin{aligned}
U(\theta, \varphi) & =e^{-i \sigma^{3} \varphi / 2} e^{-i \sigma^{2} \theta / 2} e^{i \sigma^{3} \varphi / 2} \\
& =\left(\begin{array}{cc}
\cos \frac{\theta}{2} & -\sin \frac{\theta}{2} e^{-i \varphi} \\
\sin \frac{\theta}{2} e^{i \varphi} & \cos \frac{\theta}{2}
\end{array}\right),
\end{aligned}
$$

where we should note that $U$ is a singular function on $\theta=\pi$ (the negative side of the $z$ axis) but not on $\theta=0$.

By applying the singular transformation $U$ to Eq. (2.4) we obtain, at large distances,

$$
\begin{gathered}
\Phi^{a} \rightarrow \Phi_{0}^{a}=(0,0, v)^{t}, \\
W_{i} \rightarrow W_{i}^{\text {sing. }}=U^{\dagger}\left(W_{i}-\frac{i}{g} \partial_{i}\right) U=\frac{1}{2 g r} \frac{1-\cos \theta}{\sin \theta} \hat{\varphi}_{i} \sigma^{3},
\end{gathered}
$$

with $W_{i} \equiv W_{i}^{a} \frac{\sigma^{a}}{2}$ and $\hat{\varphi}_{i}=(-\sin \varphi, \cos \varphi, 0)^{t}$. The transformed gauge field $W_{i}^{\text {sing. }}$ has a line singularity at $\theta=\pi$, which comes from the derivative $U^{\dagger} \partial_{i} U$, and hence this gauge is dubbed the singular gauge. In this gauge, the Higgs field is constant (at large distances), and thus the electromagnetic gauge field is defined straightforwardly as the $\sigma^{3}$ direction of the $S U(2)$ gauge field,

$$
\begin{aligned}
A_{i} & =\operatorname{tr}\left[\sigma^{3} W_{i}^{\text {sing. }}\right]=\frac{1}{g r} \frac{1-\cos \theta}{\sin \theta} \hat{\varphi}_{i} \\
& =\frac{1}{g}(1-\cos \theta) \partial_{i} \varphi .
\end{aligned}
$$

Let us calculate the electromagnetic field strength (2.13), which is a gauge-invariant quantity and should match with the result (2.10). The third term in Eq. (2.13) would naively seem to vanish in this gauge. However, this is not true because we have to take into account the singularity from the derivative $\partial_{i} \varphi$. Indeed, under the transformation $U$, the third term transforms as

$$
\frac{1}{g} \epsilon^{a b c} n^{a} \partial_{i} n^{b} \partial_{j} n^{c} \rightarrow-\frac{4 \pi}{g} \epsilon_{i j} \theta(-z) \delta(x) \delta(y),
$$

which describes an infinitely thin solenoid on $\theta=\pi$ (terminating at the origin), similarly to a Dirac string for a Dirac monopole. This line singularity is completely canceled by that from the gauge field (2.20), and thus we obtain the field strength as

$$
\begin{aligned}
F_{i j}^{\mathrm{EM}}= & \left(\frac{1}{g} \sin \theta \partial_{[i} \theta \partial_{j]} \varphi+\frac{1}{g}(1-\cos \theta) \partial_{[i} \partial_{j]} \varphi\right) \\
& -\frac{4 \pi}{g} \epsilon_{i j} \theta(-z) \delta(x) \delta(y) \\
= & \frac{1}{g} \epsilon_{i j k} \frac{x^{k}}{r^{3}}
\end{aligned}
$$

which is regular, coinciding with Eq. (2.10). Therefore, this configuration is an embedding of the Dirac monopole (divergenceless Dirac potential + Dirac string) into the $S U(2)$ gauge fields.

The singularity in $A_{i}$ is a gauge artifact in the sense that it does not originally exist in the hedgehog gauge. Thus, it should not be observed by a test particle with an electric charge $e=g / 2$ [spin-1/2 representation of $S U(2)]$. We thus obtain the condition that the Aharonov-Bohm (AB) effect from the singularity is not observed by the test particle,

$$
e \times q_{M}=\frac{g}{2} q_{M}=2 \pi n, \quad n \in \mathbb{Z},
$$

which is nothing but the Dirac quantization condition. Recalling that $q_{M}=4 \pi / g$, this condition is automatically satisfied with $n=1$.

We consider a more general case with a multiple topological number $m>1$ in Eq. (2.14), which provides a magnetic charge $q_{M}=4 \pi m / g$. In the singular gauge, the electromagnetic gauge field producing the magnetic charge is given by

$$
A_{i}=\frac{m}{g}(1-\cos \theta) \partial_{i} \varphi,
$$


which has also a singularity on $\theta=\pi$. The condition (2.23) leads to

$$
2 \pi m=2 \pi n, \quad n \in \mathbb{Z},
$$

which always holds with $m=n$. Therefore, the Dirac string appears in the singular gauge of the 't Hooft-Polyakov monopole, but it is always unobserved because the Dirac quantization condition is ensured by the topological quantization for $\pi_{2}\left(S^{2}\right)$.

\section{Wu-Yang monopole bundle}

A fiber bundle is a topological space consisting of a base space $B$ and a fiber $F$. The fiber bundle is locally homeomorphic to the direct product of the base space and the fiber, $B \times F$, but is not globally homeomorphic in general. A famous example is the Möbius strip, which is a nontrivial fiber bundle consisting of a circle as a base space and a segment as a fiber. Locally it seems like a cylinder, but it has an overall twist, which is only visible globally. In order to study global structures of fiber bundles it is often useful to divide the base space into several patches such that the fiber bundle is homeomorphic to the direct product on each patch. A nontrivial structure appears after gluing the patches in a nontrivial way. For the Möbius strip, we introduce two segments $[0,2 \pi] \times[-1,1]$ and glue the two ends 0 and $2 \pi$ in such a way that $(0, x) \in[0,2 \pi] \times[-1,1]$ is identified with $(2 \pi,-x) \in[0,2 \pi] \times[-1,1]$. It has a nontrivial global twist (or $\mathbb{Z}_{2}$ action: $x \rightarrow-x$ ) for the segment $[-1,1]$.

Here we discuss the Dirac quantization condition from the viewpoint of the fiber bundle. This provides us with a clear connection with the Wu-Yang description of the Dirac monopole [2], in which it is described in two patches and there is no line singularity (Dirac string) like the one seen above. Let us consider again the single monopole configuration in Eq. (2.4). We introduce a two-dimensional sphere $S^{2}$ surrounding the monopole at spatial infinity parametrized by the azimuthal angle $\varphi \in[0,2 \pi)$ and the zenith angle $\theta \in[0, \pi]$. Recall that $|\Phi|^{2}=v^{2}$ and $n^{a}=x^{a} / r$ on the sphere. To define the electromagnetic gauge field globally on the sphere without any singularities, we divide it into two patches, the hemispheres $R_{N}$ and $R_{S}$,

$$
R^{N}: 0 \leq \theta \leq \frac{\pi}{2}, \quad R^{S}: \frac{\pi}{2} \leq \theta \leq \pi,
$$

which have an overlap region on the equator $\theta=\pi / 2$, and we introduce $S U(2)$ gauge transformations defined on each hemisphere:

$$
\begin{aligned}
R^{N}: U^{N}(\theta, \varphi) & =e^{-i \sigma^{3} \varphi / 2} e^{-i \sigma^{2} \theta / 2} e^{i \sigma^{3} \varphi / 2} \\
& =\left(\begin{array}{cc}
\cos \frac{\theta}{2} & -\sin \frac{\theta}{2} e^{-i \varphi} \\
\sin \frac{\theta}{2} e^{i \varphi} & \cos \frac{\theta}{2}
\end{array}\right),
\end{aligned}
$$

$$
\begin{aligned}
R^{S}: U^{S}(\theta, \varphi) & =e^{-i \sigma^{3} \varphi / 2} e^{-i \sigma^{2}(\pi-\theta) / 2} e^{i \sigma^{3} \varphi / 2}\left(i \sigma^{2}\right) \\
& =\left(\begin{array}{cc}
e^{-i \varphi} \cos \frac{\theta}{2} & -\sin \frac{\theta}{2} \\
\sin \frac{\theta}{2} & e^{i \varphi} \cos \frac{\theta}{2}
\end{array}\right) .
\end{aligned}
$$

Both transformations bring the hedgehog-like vector $n^{a}=x^{a} / r$ into the uniform vector in each region as

$$
\begin{aligned}
& U^{N}: n^{a} \frac{\sigma^{a}}{2} \rightarrow\left(U^{N}\right)^{\dagger} n^{a} \frac{\sigma^{a}}{2} U^{N}=\frac{\sigma^{3}}{2}, \\
& U^{S}: n^{a} \frac{\sigma^{a}}{2} \rightarrow\left(U^{S}\right)^{\dagger} n^{a} \frac{\sigma^{a}}{2} U^{S}=\frac{\sigma^{3}}{2},
\end{aligned}
$$

or, equivalently, $\Phi^{a} \rightarrow \Phi_{0}^{a}=(0,0, v)$ in both $R_{N}$ and $R_{S}$. Note that $U^{N}$ and $U^{S}$ are regular on each hemisphere $R^{N}$ and $R^{S}$. On the two hemispheres, the unbroken gauge group $U(1)_{\mathrm{EM}}$ is uniformly defined as the $\sigma^{3}$ subgroup of $S U(2)$.

Since we have $U^{N} \Phi=U^{S} \Phi=(0,0, v)^{t}$ in the overlap region of the two patches, $\theta=\pi / 2$, the transition function $\left(U^{S}\right)^{-1} U^{N}$ must leave $\Phi$ invariant, i.e., $\left(U^{S}\right)^{-1} U^{N} \in$ $U(1)_{\mathrm{EM}}$. Indeed, we have

$$
\left(U^{S}\right)^{-1} U^{N}=e^{i \varphi \sigma^{3}}
$$

and thus it does not change $\Phi_{0}^{a}=(0,0, v)^{t}$. The transition function is a map from the equator $S^{1}: \varphi \in[0,2 \pi)$ into $U(1)_{\mathrm{EM}}\left(\simeq S^{1}\right)$ with a winding number of unity, and hence the two patches are glued in a topologically nontrivial way.

Let us define the electromagnetic gauge field after the transformations in Eqs. (2.29) and (2.30) on each patch $R^{N}$ and $R^{S}$ as

$$
\begin{gathered}
A_{i}^{N} \equiv W_{i}^{3}=\frac{1}{g} \frac{1-\cos \theta}{r \sin \theta} \hat{\varphi}_{i}, \\
A_{i}^{S} \equiv W_{i}^{3}=-\frac{1}{g} \frac{1+\cos \theta}{r \sin \theta} \hat{\varphi}_{i} .
\end{gathered}
$$

Importantly, they are regular on each patch, as desired. On the equator $\theta=\pi / 2$ they differ by the $U(1)_{\mathrm{EM}}$ gauge transformation:

$$
A_{i}^{N}=A_{i}^{S}+\frac{2}{g r} \hat{\varphi}_{i} \equiv A_{i}^{S}+\Delta A_{i} .
$$

The difference $\Delta A_{i}$ originates from the transition function (2.31) as

$$
\Delta A_{i} \frac{\sigma^{3}}{2}=\frac{-i}{g}\left[\left(U^{S}\right)^{-1} U^{N}\right]^{\dagger} \partial_{i}\left[\left(U^{S}\right)^{-1} U^{N}\right] .
$$

We introduce the first Chern number, which characterizes the topology of the fiber bundle with the fiber $U(1)_{\mathrm{EM}}$ over the base space $S^{2}$ surrounding the monopole, as 


$$
\int_{S^{2}} c_{1} \equiv \frac{e}{2 \pi} \int_{S^{2}} F^{\mathrm{EM}}
$$

where $c_{1}$ is called the first Chern class and $S^{2}$ consists of $R^{N}$ and $R^{S}$. The magnetic charge of the monopole coincides with the first Chern number up to an overall constant. Substituting Eqs. (2.32) and (2.33) into Eq. (2.36), we have

$$
\begin{aligned}
\int_{S^{2}} c_{1} & =\frac{g}{4 \pi} \int_{S^{1}: \theta=\pi / 2}\left(A^{N}-A^{S}\right)=\frac{g}{4 \pi} \int_{S^{1}} \Delta A \\
& =\frac{1}{2 \pi} \int_{0}^{2 \pi} d \varphi=1,
\end{aligned}
$$

where $F^{\mathrm{EM}} \equiv \frac{1}{2} F_{i j}^{\mathrm{EM}} d x^{i} \wedge d x^{j}, A^{N(S)} \equiv A_{i}^{N(S)} d x^{i}$ and we have used the definition of the electric charge $e \equiv g / 2$ and Stokes' theorem. From Eq. (2.37), the first Chern number counts the winding number of the transition function on the equator, and the nonzero value means that the two patches $R^{N}$ and $R^{S}$ are nontrivially glued. Thus, the transition function is in a nontrivial topological sector classified by the first homotopy group $\pi_{1}\left(S^{1}\right)=\mathbb{Z}$. In the language of fiber bundles, this means that the topological space consisting of the base space $S^{2}$ and the fiber $U(1)_{\mathrm{EM}}$ is nontrivial [not $S^{2} \times U(1)_{\mathrm{EM}}$ ] and is homeomorphic to $S^{3}$ (the Hopf fibration). Therefore, the configuration in Eqs. (2.32) and (2.33) is regarded as an embedding of the Wu-Yang description of the Dirac monopole into the $S U(2)$ gauge theory.

In this formulation, the Dirac quantization condition can be expressed as the single valuedness of a wave function of a test particle with an electric charge $e$. When the test particle goes once around the equator $\theta=\pi / 2$, it receives the $\mathrm{AB}$ phase

$$
\theta_{A B}=e \oint d x_{i} A_{i}
$$

In order for the wave function to be single valued, the two effects calculated on the two patches $R^{N}$ and $R^{S}$ must be equivalent. Thus, we obtain the condition

$$
\begin{gathered}
e \oint_{\theta=\pi / 2} d x_{i} \Delta A_{i}=2 \pi n(n \in \mathbb{Z}) \\
\Leftrightarrow 2 \pi \int_{S^{2}} c_{1}=2 \pi n,
\end{gathered}
$$

which is automatically satisfied with $n=1$ by Eq. (2.38). This result can be extended to more general cases with monopole charges larger than unity.

In this section we have looked at the 't Hooft-Polyakov monopole in two ways: in the singular gauge and in the language of fiber bundles. While the former corresponds to the Dirac monopole and the Dirac string, the latter is related to the Wu-Yang description without the Dirac string. Both provide the Dirac quantization condition, but it is automatically satisfied due to the nontrivial second or first homotopy groups, $\pi_{2}(G / H)=\mathbb{Z}$ or $\pi_{1}\left(S^{1}\right)=\mathbb{Z}$. In the following sections, we study these for a Nambu monopole in the SM and 2HDM.

\section{NAMBU MONOPOLE IN THE STANDARD MODEL}

\section{A. Hedgehog gauge}

Here we discuss the Nambu monopole [12] in the SM in the hedgehog gauge. The gauge-Higgs sector of the SM Lagrangian is

$$
\begin{aligned}
\mathcal{L}= & -\frac{1}{4} W_{\mu \nu}^{a} W^{a \mu \nu}-\frac{1}{4} Y_{\mu \nu} Y^{\mu \nu}+\left|D_{\mu} \Phi_{\mathrm{SM}}\right|^{2} \\
& -\lambda\left(\left|\Phi_{\mathrm{SM}}\right|^{2}-v^{2}\right)^{2}
\end{aligned}
$$

where $\Phi_{\mathrm{SM}}$ is the SM Higgs doublet. The covariant derivative is $D_{\mu} \Phi_{\mathrm{SM}}=\left(\partial_{\mu}-i \frac{g}{2} W_{\mu}^{a} \sigma^{a}-i \frac{g^{\prime}}{2} Y_{\mu}\right) \Phi_{\mathrm{SM}}$, where $W$ and $Y$ are the $S U(2)_{W}$ and $U(1)_{Y}$ gauge fields, respectively.

The Nambu monopole, which is a magnetic monopole attached by a $Z$ string ( $Z$-flux tube), was first formulated by Nambu [12]. Since the electroweak symmetry breaking $S U(2)_{W} \times U(1)_{Y} \rightarrow U(1)_{\mathrm{EM}}$ is topologically trivial, neither the Nambu monopole nor the $Z$ string can be topologically stable. We consider a situation where the monopole lies on the origin and the $Z$ string emanating from it is put on the positive side of the $z$ axis $(\theta=0)$. At large distances from the monopole, $r \rightarrow \infty$, the configuration describing the monopole is given by

$$
\begin{gathered}
\Phi_{\mathrm{SM}}=v\left(\begin{array}{c}
e^{i \varphi} f(\theta) \cos \frac{\theta}{2} \\
\sin \frac{\theta}{2}
\end{array}\right), \\
g W_{i}^{a}=-\cos ^{2} \theta_{W} \frac{x^{a}}{r} h(\theta)(1+\cos \theta) \partial_{i} \varphi \\
-\epsilon^{a b c} \frac{x^{b}}{r} \partial_{i} \frac{x^{c}}{r}, \\
g^{\prime} Y_{i}=-\sin ^{2} \theta_{W} j(\theta)(1+\cos \theta) \partial_{i} \varphi,
\end{gathered}
$$

where $\theta_{W}$ is the Weinberg angle. Here $f(\theta), h(\theta)$, and $j(\theta)$ are profile functions that vanish on $\theta=0$ (the positive side of the $z$ axis),

$$
f(0)=h(0)=j(0)=0,
$$

and thus the electroweak symmetry is restored $\left(\left|\Phi_{\mathrm{SM}}\right|=0\right)$ on $\theta=0$ corresponding to the $Z$ string (string-like defect).

As we move away from $\theta=0$ on the large sphere, the profile functions approach unity, and then we obtain the following asymptotic forms: 


$$
\begin{gathered}
\Phi_{\mathrm{SM}}=v\left(\begin{array}{c}
e^{i \varphi} \cos \frac{\theta}{2} \\
\sin \frac{\theta}{2}
\end{array}\right), \\
g W_{i}^{a}=-\cos ^{2} \theta_{W} n^{a}(1+\cos \theta) \partial_{i} \varphi-\epsilon^{a b c} \frac{x^{b}}{r} \partial_{i} \frac{x^{c}}{r}, \\
g^{\prime} Y_{i}=-\sin ^{2} \theta_{W}(1+\cos \theta) \partial_{i} \varphi .
\end{gathered}
$$

These configurations seem to have a line singularity on $\theta=0$ corresponding to the $Z$ string and thus are valid only outside of the string. Note that the $Z$ string is a regular and physical object, unlike the Dirac string.

We introduce a unit vector as

$$
n_{\mathrm{SM}}^{a} \equiv \frac{\Phi_{\mathrm{SM}}^{\dagger} \sigma^{a} \Phi_{\mathrm{SM}}}{\left|\Phi_{\mathrm{SM}}\right|^{2}}
$$

transforming as the adjoint representation of $S U(2)_{W}$, which is analogous to that of the 't Hooft-Polyakov monopole (2.5). Substituting Eqs. (3.6)-(3.8), we have ${ }^{4}$

$$
\begin{aligned}
n_{\mathrm{SM}}^{a} & =\frac{1}{f^{2} c_{1 / 2}^{2}+s_{1 / 2}^{2}}\left(f \frac{x}{r}, f \frac{y}{r}, f^{2} c_{1 / 2}^{2}-s_{1 / 2}^{2}\right) \\
& =\left(\sin \Theta_{\mathrm{SM}} \cos \varphi, \sin \Theta_{\mathrm{SM}} \sin \varphi, \cos \Theta_{\mathrm{SM}}\right),
\end{aligned}
$$

where we have defined

$$
s_{1 / 2} \equiv \sin \frac{\theta}{2}, \quad c_{1 / 2} \equiv \cos \frac{\theta}{2}
$$

and

$$
\begin{gathered}
\sin \Theta_{\mathrm{SM}} \equiv \frac{f}{f^{2} c_{1 / 2}^{2}+s_{1 / 2}^{2}} \frac{\sqrt{x^{2}+y^{2}}}{r}, \\
\cos \Theta_{\mathrm{SM}} \equiv \frac{f^{2} c_{1 / 2}^{2}-s_{1 / 2}^{2}}{f^{2} c_{1 / 2}^{2}+s_{1 / 2}^{2}} .
\end{gathered}
$$

Note that $n_{\mathrm{SM}}^{a}$ is singular at $\theta=0$ because the electroweak gauge symmetry is restored at $\theta=0\left(\left|\Phi_{\mathrm{SM}}\right|=0\right)$.

We can define a $U(1)$ subgroup of $S U(2)_{W}$ that keeps the vector $n_{\text {SM }}^{a}$ invariant, which is denoted by $U(1)_{n}{ }^{5}$ The electromagnetic and $Z$ field strengths are defined as superpositions of $U(1)_{n}$ and $U(1)_{Y}$ [12]:

$$
\begin{gathered}
F_{i j}^{\mathrm{EM}} \equiv-\sin \theta_{W} n_{\mathrm{SM}}^{a} W_{i j}^{a}+\cos \theta_{W} Y_{i j}, \\
F_{i j}^{Z} \equiv-\cos \theta_{W} n_{\mathrm{SM}}^{a} W_{i j}^{a}-\sin \theta_{W} Y_{i j} .
\end{gathered}
$$

\footnotetext{
${ }^{4}$ For the Higgs field to be regular at the center of the string, $\rho=0$, the function $f(\theta)$ must satisfy $f(\theta) \sim \sin \theta$ for $\theta \rightarrow 0$ $[16,61,62]$.

${ }^{5}$ Although $U(1)_{n}$ keeps $n_{\text {SM }}^{a}$ invariant, it does not keep $\Phi_{\mathrm{SM}}$ invariant, and thus $U(1)_{n}$ itself is not an unbroken subgroup.
}

From the fact that we cannot define the subgroup $U(1)_{n}$ at $\theta=0$ because $n_{\text {SM }}^{a}$ is ill defined there, it follows that the electromagnetic and $Z$ field strengths (3.14) and (3.15) are not defined at $\theta=0$. This is an important difference from the 't Hooft-Polyakov case.

To see the electromagnetic and $Z$ fluxes at large distances from the string core, it is convenient to use the asymptotic forms in Eqs. (3.6)-(3.8) at $\theta \gg 0$. From Eq. (3.6), we can see that $n_{\text {SM }}^{a}$ approaches a hedgehog structure,

$$
n_{\mathrm{SM}}^{a}=(\sin \theta \cos \varphi, \sin \theta \sin \varphi, \cos \theta)^{t}=\frac{x^{a}}{r} .
$$

Plugging this and the ansatz in Eqs. (3.6)-(3.8) into Eqs. (3.14) and (3.15), we get the physical field strengths at large distances as

$$
\begin{gathered}
F_{i j}^{Z}=\frac{4 \pi \cos \theta_{\mathrm{W}}}{g} \theta(z) \epsilon_{3 i j} \delta(x) \delta(y), \\
F_{i j}^{\mathrm{EM}}=\frac{\sin \theta_{\mathrm{W}}}{g} \epsilon^{a i j} \frac{x^{a}}{r^{3}} .
\end{gathered}
$$

Note that the $\delta$-function singularity at $\theta=0$ in $F_{i j}^{Z}$ comes from the asymptotic forms at $\theta \gg 0$ and the true form has a finite width (the $Z$ string is a regular solution), decaying exponentially like $\sim \exp \left(-m_{Z} \rho\right)$. From Eq. (3.18), it is clear that there is a magnetic flux from the origin in a spherical hedgehog form. The total amount of the magnetic flux $\Phi_{\mathrm{EM}}$ can be calculated by integrating the flux density $B_{i} \equiv \frac{1}{2} \epsilon_{i j k} F_{j k}^{\mathrm{EM}}$ as

$$
\Phi_{\mathrm{EM}}=\int d^{3} x \partial_{i} B_{i}=\frac{4 \pi \sin \theta_{\mathrm{W}}}{g} .
$$

In addition, from Eq. (3.17) the $Z$ fluxes only exist on the positive side of the $z$ axis as

$$
\left.\Phi_{Z}\right|_{z>0}=\left.\int d^{2} x F_{i j}^{Z}\right|_{z>0}=\frac{4 \pi \cos \theta_{\mathrm{W}}}{g},
$$

which flows on the $z$ axis from the origin. Therefore, the former indeed yields a long-range force, while the latter is massive and confined around the string on $\theta=0$. See Fig. 1 for a schematic picture of the $Z$ and electromagnetic fluxes for the Nambu monopole in the SM.

\section{B. Fiber bundle}

To clarify a (non)topological property of the Nambu monopole in the SM, let us discuss the fiber bundle of the electromagnetic gauge group $U(1)_{\mathrm{EM}}$. We consider a twodimensional sphere $S^{2}$ surrounding the monopole at spatial infinity, where the single $Z$ string passes through the sphere (see Fig. 1). Here we make an important remark: the vector $n_{\mathrm{SM}}^{a}$ indicating the subgroup $U(1)_{n}$ of $S U(2)_{W}$ is ill defined 


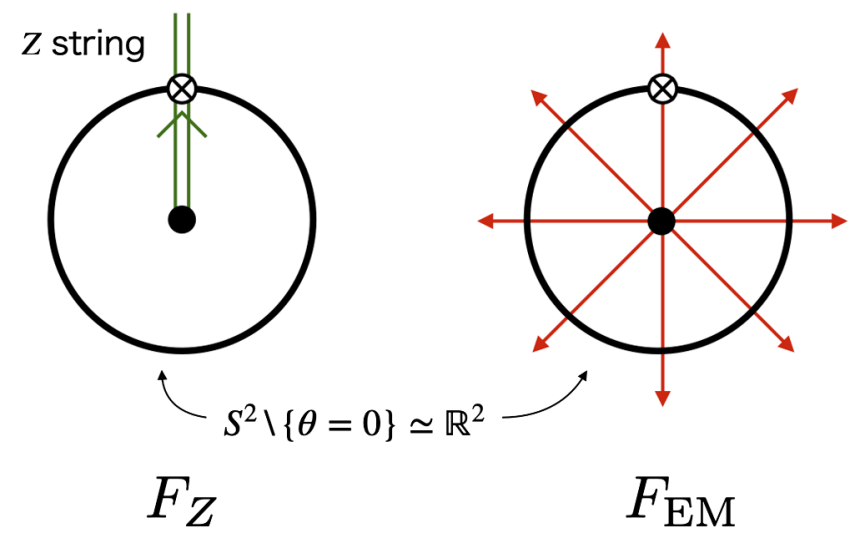

FIG. 1. Schematic picture of $S^{2}$ surrounding the Nambu monopole in the SM. The $Z$ string passes through the north pole on $S^{2}$, and makes the electromagnetic gauge field singular at the string core $\theta=0$. As a result, it is well defined only on $S^{2} \backslash\{\theta=0\} \simeq \mathbb{R}^{2}$. Such a base space can be spanned by a single patch.

at the string center $\theta=0$. As a result, we cannot define $U(1)_{n}$ and hence $U(1)_{\mathrm{EM}}$ at the north pole $\theta=0$ on $S^{2}$. Thus, we must consider the base space $S^{2} \backslash\{\theta=0\} \simeq \mathbb{R}^{2}$ with the fiber $U(1)_{\mathrm{EM}}$, instead of $S^{2}$. Obviously, we can span $\mathbb{R}^{2}$ by a single patch and the fiber bundle is trivial: $\mathbb{R}^{2} \times U(1)_{\mathrm{EM}}\left(\simeq \mathbb{R}^{2} \times S^{1}\right)$. Therefore, this configuration is topologically distinct from the previous case of the 't Hooft-Polyakov monopole, and its topological structure is trivial. As a result of the fact that the monopole has no topological origin, i.e., the trivial fiber bundle $\mathbb{R}^{2} \times U(1)_{\mathrm{EM}}$, the monopole does not provide the Dirac quantization condition, and thus the magnetic charge is not quantized. Indeed, the monopole can be removed by a continuous deformation; for instance, we can deform the $Z$ string into the vacuum since the $Z$ string is not topologically protected $\left[\pi_{1}\left(S^{3}\right)=0\right.$ for the vacuum manifold $S^{3}$ of the SM], and hence the monopole disappears.

\section{DEFINITION OF THE ELECTROMAGNETIC FIELD IN THE 2HDM}

\section{A. Two-Higgs-doublet model}

Before turning to the Nambu monopole in the 2HDM, here we clarify the definition of the electromagnetic gauge group in the $2 \mathrm{HDM}$. In a homogeneous vacuum in the absence of any solitons, we can obviously define the electromagnetic field and the field strength by a projection of the gauge fields onto the unbroken direction. On the other hand, it is nontrivial in the presence of solitons, in which the Higgs fields, i.e., the unbroken direction of the gauge symmetry, varies in the space. The definition without solitons should be replaced by a more general definition for the cases with varying vacuum expectation values (VEVs). In the SM, the electromagnetic field strength is defined by Eq. (3.14), which was given by Nambu in Ref. [12]. There are also other definitions (e.g., those in Refs. [63,64]), although they are equivalent at large distances from the solitons, in which $D_{i} \Phi_{\mathrm{SM}} \sim 0$ and $\left|\Phi_{\mathrm{EM}}\right| \sim v$. In the $2 \mathrm{HDM}$, on the other hand, general definitions for the electromagnetic field that can be applied to the varying VEVs have not been given in the literature. For later use, we present such a general definition of the electromagnetic field in the presence of solitons in the 2HDM for the first time.

We start with the Lagrangian of the electroweak sector in the 2HDM, in which there are two Higgs doublets $\Phi_{1}$ and $\Phi_{2}$ with the same $U(1)_{Y}$ hypercharge +1 . The Lagrangian is given by

$\mathcal{L}=-\frac{1}{4} W_{\mu \nu}^{a} W^{a \mu \nu}-\frac{1}{4} Y_{\mu \nu} Y^{\mu \nu}+\operatorname{Tr}\left|D_{\mu} H\right|^{2}-V(H)$,

where $W_{\mu}$ and $Y_{\mu}$ are the $S U(2)_{W}$ and $U(1)_{Y}$ gauge fields, respectively. We have adopted the $2 \times 2$ matrix notation, $H \equiv\left(i \sigma_{2} \Phi_{1}^{*}, \Phi_{2}\right)[65]$. The matrix field $H$ transforms under the electroweak $S U(2)_{W} \times U(1)_{Y}$ symmetry as

$$
H \rightarrow \exp \left[\frac{i}{2} \theta_{a}(x) \sigma_{a}\right] H \exp \left[-\frac{i}{2} \theta_{Y}(x) \sigma_{3}\right],
$$

where the group element acting from the left belongs to $S U(2)_{W}$ and the other element acting from the right belongs to $U(1)_{Y}$. Therefore, the covariant derivative on $H$ can be expressed as

$$
D_{\mu} H=\partial_{\mu} H-i \frac{g}{2} \sigma_{a} W_{\mu}^{a} H+i \frac{g^{\prime}}{2} H \sigma_{3} Y_{\mu}
$$

The VEVs of $H$ are expressed by a diagonal matrix $\langle H\rangle=\operatorname{diag}\left(v_{1}, v_{2}\right)$, and the Higgs potential can be written by using $H$ as follows:

$$
\begin{aligned}
V(H)= & -m_{1}^{2} \operatorname{Tr}|H|^{2}-m_{2}^{2} \operatorname{Tr}\left(|H|^{2} \sigma_{3}\right)-\left(m_{3}^{2} \operatorname{det} H+\text { H.c. }\right) \\
& +\alpha_{1} \operatorname{Tr}|H|^{4}+\alpha_{2}\left(\operatorname{Tr}|H|^{2}\right)^{2}+\alpha_{3} \operatorname{Tr}\left(|H|^{2} \sigma_{3}|H|^{2} \sigma_{3}\right) \\
& +\alpha_{4} \operatorname{Tr}\left(|H|^{2} \sigma_{3}|H|^{2}\right)+\left(\alpha_{5} \operatorname{det} H^{2}+\text { H.c. }\right),
\end{aligned}
$$

where $|H|^{2} \equiv H^{\dagger} H$ and we have imposed a (softly broken) $\mathbb{Z}_{2}$ symmetry, $\Phi_{1} \rightarrow+\Phi_{1}, \Phi_{2} \rightarrow-\Phi_{2}$ (or, equivalently, $\left.H \rightarrow H \sigma_{3}\right)$. The relations between the parameters in Eq. (4.4) and the conventional parametrization were shown in Ref. [59].

In the next section, we will consider the topologically stable Nambu monopole in the 2HDM. The stability is realized by setting $m_{3}=\alpha_{4}=\alpha_{5}=0$ and $\alpha_{3}<0$ in the potential [59], resulting in two global symmetries: 


$$
\begin{cases}U(1)_{a}: & H \rightarrow e^{i \alpha} H, \\ \left(\mathbb{Z}_{2}\right)_{\mathrm{C}}: & H \rightarrow\left(i \sigma_{2}\right)^{\dagger} H\left(i \sigma_{2}\right), \quad W_{\mu} \rightarrow\left(i \sigma_{2}\right)^{\dagger} W_{\mu}\left(i \sigma_{2}\right), \quad Y_{\mu} \rightarrow-Y_{\mu} .\end{cases}
$$

As a result of the $\left(\mathbb{Z}_{2}\right)_{C}$ symmetry, we have $v_{1}=v_{2}$ $\left(\tan \beta \equiv v_{2} / v_{1}=1\right)^{6}$

Using the two doublets, we define two unit vectors

$$
n_{1}^{a} \equiv \frac{\Phi_{1}^{\dagger} \sigma^{a} \Phi_{1}}{\left|\Phi_{1}\right|^{2}}, \quad n_{2}^{a} \equiv \frac{\Phi_{2}^{\dagger} \sigma^{a} \Phi_{2}}{\left|\Phi_{2}\right|^{2}}
$$

which are analogous to Eq. (3.9) in the SM and are ill defined when $\left|\Phi_{1}\right|=0$ and $\left|\Phi_{2}\right|=0$, respectively.

\section{B. $n_{1}^{a}=n_{2}^{a}$ case}

We first assume that $n_{1}^{a}=n_{2}^{a}$ holds in regions where $\Phi_{1} \neq 0$ and $\Phi_{2} \neq 0$, which we will encounter in the subsequent sections. Taking the unitary gauge, this situation is realized when the doublets have the following forms:

$$
\Phi_{1}=\left(\begin{array}{c}
0 \\
\Phi_{1,2}
\end{array}\right), \quad \Phi_{2}=\left(\begin{array}{c}
0 \\
\Phi_{2,2}
\end{array}\right)
$$

where $\Phi_{1,2}$ and $\Phi_{2,2}$ are complex functions. This configuration is sometimes called the neutral configuration in the literature. We will relax this condition later.

In the presence of solitons, the Higgs fields $H$ acquire $x$-dependent VEVs, and then the gauge fields obtain $x$-dependent masses as follows:

$$
\begin{aligned}
\operatorname{Tr}\left|D_{\mu} H\right|^{2} & =\left|D_{\mu} \Phi_{1}\right|^{2}+\left|D_{\mu} \Phi_{2}\right|^{2} \\
& \supset \sum_{f=1,2}\left|\left(-\frac{i}{2} g W_{\mu}^{a} \sigma^{a}-\frac{i}{2} g^{\prime} Y_{\mu}\right) \Phi_{f}\right|^{2} \\
& =\frac{1}{4} \sum_{f} \Phi_{f}^{\dagger}\left(g^{2} W_{\mu}^{a} W^{b \mu} \sigma^{a} \sigma^{b}+g^{\prime 2} Y_{\mu} Y^{\mu}+2 g g^{\prime} W_{\mu}^{a} Y^{\mu} \sigma^{a}\right) \Phi_{f} \\
& =\frac{1}{4} \sum_{f}\left|\Phi_{f}\right|^{2}\left(g^{2} W_{\mu}^{a} W^{a \mu}+g^{2} Y_{\mu} Y^{\mu}\right)+2 g g^{\prime} \frac{1}{4} \sum_{f}\left|\Phi_{f}\right|^{2} n_{f}^{a} W_{\mu}^{a} Y^{\mu} .
\end{aligned}
$$

In order to rewrite Eq. (4.8), we introduce an adjoint unit vector $n^{a}$ as

$$
\begin{aligned}
\sum_{f}\left|\Phi_{f}\right|^{2} n_{f}^{a} & \equiv n^{a} \sum_{f}\left|\Phi_{f}\right|^{2} \\
\Leftrightarrow n^{a} & \equiv \frac{\Phi_{1}^{\dagger} \sigma^{a} \Phi_{1}+\Phi_{2}^{\dagger} \sigma^{a} \Phi_{2}}{\left|\Phi_{1}\right|^{2}+\left|\Phi_{2}\right|^{2}}=\frac{\sum_{f}\left|\Phi_{f}\right|^{2} n_{f}^{a}}{\sum_{f}\left|\Phi_{f}\right|^{2}},
\end{aligned}
$$

which is well defined everywhere except for $\left|\Phi_{1}\right|=\left|\Phi_{2}\right|=0$. We thus have

$$
\begin{aligned}
(4.8)= & \frac{1}{4} \sum_{f}\left|\Phi_{f}\right|^{2}\left(g n^{a} W_{\mu}^{a}+g^{\prime} Y_{\mu}\right)^{2} \\
& +\frac{g^{2}}{4} \sum_{f}\left|\Phi_{f}\right|^{2}\left(\delta^{a b}-n^{a} n^{b}\right) W_{\mu}^{a} W_{\mu}^{b} \\
\equiv & \frac{g_{Z}^{2}}{4} \sum_{f}\left|\Phi_{f}\right|^{2} Z_{\mu} Z^{\mu}+\frac{g^{2}}{4} \sum_{f}\left|\Phi_{f}\right|^{2} \operatorname{Tr}\left(W_{\mu}^{\perp} W^{\perp \mu}\right),
\end{aligned}
$$

\footnotetext{
${ }^{6}$ The reader should not confuse the $\left(\mathbb{Z}_{2}\right)_{\mathrm{C}}$ symmetry with the softly broken $\mathbb{Z}_{2}$ symmetry: $\Phi_{1} \rightarrow+\Phi_{1}, \Phi_{2} \rightarrow-\Phi_{2}$.
}

where we have defined

$$
\begin{gathered}
Z_{\mu} \equiv-\cos \theta_{W} n^{a} W_{\mu}^{a}-\sin \theta_{W} Y_{\mu}, \\
W_{\mu}^{\perp} \equiv \frac{\sigma^{a}}{2}\left(\delta_{a b}-n_{a} n_{b}\right) W_{\mu}^{b},
\end{gathered}
$$

and $g_{Z} \equiv \sqrt{g^{2}+g^{\prime 2}}$. Equations (4.11) and (4.12) are the neutral $Z$ boson and the charged $W$ boson, respectively. ${ }^{7}$ On the other hand, the massless electromagnetic gauge field (photon) is defined by

$$
A_{\mu} \equiv-\sin \theta_{W} n^{a} W_{\mu}^{a}+\cos \theta_{W} Y_{\mu},
$$

which is orthogonal to the $Z$ field. We stress that the electromagnetic and massive gauge bosons can be defined everywhere unless $\Phi_{1}=\Phi_{2}=0$.

Similarly, we can define the electromagnetic and $Z$ field strengths using $n^{a}$ as

$$
F_{\mu \nu}^{Z} \equiv-\cos \theta_{W} n^{a} W_{\mu \nu}^{a}-\sin \theta_{W} Y_{\mu \nu}
$$

\footnotetext{
${ }^{7}$ Note that $W_{\mu}^{\perp}$ itself is not an eigenstate of $U(1)_{\mathrm{EM}}$.
} 


$$
F_{\mu \nu}^{\mathrm{EM}} \equiv-\sin \theta_{W} n^{a} W_{\mu \nu}^{a}+\cos \theta_{W} Y_{\mu \nu}
$$

respectively. Indeed, the latter satisfies the source-free Maxwell equations

$$
\partial^{\mu} F_{\mu \nu}^{\mathrm{EM}}=0, \quad \partial^{\mu} \tilde{F}_{\mu \nu}^{\mathrm{EM}}=0
$$

at large distances, and yields the long-range force. Note that these definitions are analogous to those in the SM, but $n_{\mathrm{SM}}^{a}$ is replaced by $n^{a}$ [Eq. (4.9)].

\section{C. $n_{1}^{a} \neq n_{2}^{a}$ case}

We consider the case of $n_{1}^{a} \neq n_{2}^{a}$. In the unitary gauge, this is realized when, e.g.,

$$
\Phi_{1}=\left(\begin{array}{c}
0 \\
\Phi_{1,2}
\end{array}\right), \quad \Phi_{2}=\left(\begin{array}{c}
\Phi_{2,1} \\
\Phi_{2,2}
\end{array}\right),
$$

where $\Phi_{1,2}, \Phi_{2,1}$, and $\Phi_{2,2}$ are complex functions. In the literature, this is sometimes called the charged configuration. In such a case, there is no unbroken subgroup in the $S U(2)_{W} \times U(1)_{Y}$ symmetry, and thus $U(1)_{\mathrm{EM}}$ is also broken. To see this, we start with the mass terms for the gauge fields again:

$$
\begin{aligned}
\operatorname{Tr}\left|D_{\mu} H\right|^{2} \supset & \sum_{f=1,2}\left|\left(-\frac{i}{2} g W_{\mu}^{a} \sigma^{a}-\frac{i}{2} g^{\prime} Y_{\mu}\right) \Phi_{f}\right|^{2} \\
= & \frac{1}{4} \sum_{f}\left|\Phi_{f}\right|^{2}\left(g^{2} W_{\mu}^{a} W^{a \mu}+g^{\prime 2} Y_{\mu} Y^{\mu}\right) \\
& +2 g g^{\prime} \frac{1}{4} \sum_{f}\left|\Phi_{f}\right|^{2} n_{f}^{a} W_{\mu}^{a} Y^{\mu} .
\end{aligned}
$$

Let us rewrite the last term in Eq. (4.18). Note that the vector $n^{a}$ defined in Eq. (4.9) is no longer normalized to unity because of $n_{1}^{a} \neq n_{2}^{a}$. Thus, we introduce an alternative unit vector $\tilde{n}^{a}$ as

$$
\begin{aligned}
\sum_{f}\left|\Phi_{f}\right|^{2} n_{f}^{a} & \equiv C \tilde{n}^{a} \\
\Leftrightarrow \tilde{n}^{a} & \equiv \frac{\Phi_{1}^{\dagger} \sigma^{a} \Phi_{1}+\Phi_{2}^{\dagger} \sigma^{a} \Phi_{2}}{C},
\end{aligned}
$$

where $C$ is a normalization factor,

$$
C^{2}=\left(\Phi_{1}^{\dagger} \sigma^{a} \Phi_{1}+\Phi_{2}^{\dagger} \sigma^{a} \Phi_{2}\right)^{2}
$$

and is taken as $C>0$.

Using Eq. (4.19) and dividing $\left(W_{\mu}^{a}\right)^{2}$ into two parts, we can rewrite Eq. (4.18) as

$$
\begin{aligned}
(4.18)= & \frac{1}{4} \sum_{f}\left|\Phi_{f}\right|^{2} g^{2}\left(\tilde{n}^{a} W_{\mu}^{a}\right)^{2}+\frac{1}{4} \sum_{f}\left|\Phi_{f}\right|^{2} g^{2}\left(Y_{\mu}\right)^{2} \\
& +2 g g^{\prime} \frac{1}{4} C \tilde{n}^{a} W_{\mu}^{a} Y^{\mu} \\
& +\frac{g^{2}}{4} \sum_{f}\left|\Phi_{f}\right|^{2}\left(\delta^{a b}-\tilde{n}^{a} \tilde{n}^{b}\right) W_{\mu}^{a} W^{b \mu} \\
= & \frac{1}{4}\left(\begin{array}{c}
\tilde{n}^{a} W_{\mu}^{a} \\
Y_{\mu}
\end{array}\right)^{T} M\left(\begin{array}{c}
\tilde{n}^{a} W_{\mu}^{a} \\
Y_{\mu}
\end{array}\right) \\
& +\frac{g^{2}}{4} \sum_{f}\left|\Phi_{f}\right|^{2} \operatorname{Tr}\left(W_{\mu}^{\perp} W^{\perp \mu}\right),
\end{aligned}
$$

with

$$
M \equiv\left(\begin{array}{cc}
g^{2} \sum_{f}\left|\Phi_{f}\right|^{2} & g g^{\prime} C \\
g g^{\prime} C & g^{\prime 2} \sum_{f}\left|\Phi_{f}\right|^{2}
\end{array}\right)
$$

and

$$
W_{\mu}^{\perp} \equiv \frac{\sigma^{a}}{2}\left(\delta^{a b}-\tilde{n}^{a} \tilde{n}^{b}\right) W_{\mu}^{b},
$$

where $W_{\mu}^{\perp}$ is the orthogonal component to $\tilde{n}^{a}$, similarly to Eq. (4.12).

The mass matrix $M$ in Eq. (4.22) provides masses for the $Z$ gauge boson and photon. Unlike the previous case, the matrix have two nonzero eigenvalues, and thus there is no massless field. However, we can still define a photon as the lighter component among the two massive gauge bosons. To achieve this, let us diagonalize the mass matrix $M$ by the following basis transformation:

$$
\left(\begin{array}{c}
\tilde{n}^{a} W_{\mu}^{a} \\
Y_{\mu}
\end{array}\right) \rightarrow U\left(\begin{array}{c}
\tilde{n}^{a} W_{\mu}^{a} \\
Y_{\mu}
\end{array}\right)
$$

with

$$
U \equiv\left(\begin{array}{cc}
\cos \xi & \sin \xi \\
-\sin \xi & \cos \xi
\end{array}\right)
$$

where $\xi$ is an "effective Weinberg angle" satisfying

$$
\tan 2 \xi=r_{C} \tan 2 \theta_{W},
$$

with $r_{C} \equiv \sum_{f}\left|\Phi_{f}\right|^{2} / C$.

The mass eigenvalues and mass eigenstates are

$\frac{1}{4}\left(\begin{array}{c}\tilde{n}^{a} W_{\mu}^{a} \\ Y_{\mu}\end{array}\right)^{T} M\left(\begin{array}{c}\tilde{n}^{a} W_{\mu}^{a} \\ Y_{\mu}\end{array}\right)=\frac{m_{Z}^{2}}{2} Z_{\mu} Z^{\mu}+\frac{m_{\gamma}^{2}}{2} A_{\mu} A^{\mu}$,

with 
$m_{Z}^{2} \equiv \frac{\sum_{f}\left|\Phi_{f}\right|^{2}}{4}\left(1+\cos 2 \theta_{W} \cos 2 \xi+r_{C} \sin 2 \theta_{W} \sin 2 \xi\right)$,

$m_{\gamma}^{2} \equiv \frac{\sum_{f}\left|\Phi_{f}\right|^{2}}{4}\left(1-\cos 2 \theta_{W} \cos 2 \xi-r_{C} \sin 2 \theta_{W} \sin 2 \xi\right)$,

and

$$
\begin{aligned}
& Z_{\mu} \equiv-\cos \xi \tilde{n}^{a} W_{\mu}^{a}-\sin \xi Y_{\mu}, \\
& A_{\mu} \equiv-\sin \xi \tilde{n}^{a} W_{\mu}^{a}+\cos \xi Y_{\mu} .
\end{aligned}
$$

Note that $A_{\mu}$ is the electromagnetic gauge field (photon), but it is no longer massless, $m_{\gamma} \neq 0$. For arbitrary $\theta_{W}$ and $C \neq 0, m_{\gamma}^{2}<m_{Z}^{2}$ always holds.

To compare with the previous result in the last subsection, let us reproduce the case of $n_{1}^{a}=n_{2}^{a}$. It leads to $n^{a}=\tilde{n}^{a}$, and hence $r_{C}=1$ and $\xi=\theta_{W}$. The mass eigenstates (4.30) and (4.31) reduce to the previous result, Eqs. (4.11) and (4.13). The mass eigenvalues (4.28) and (4.29) reduce to

$$
m_{Z}^{2} \rightarrow \frac{\sum_{f}\left|\Phi_{f}\right|^{2}}{2}, \quad m_{\gamma}^{2} \rightarrow 0
$$

which agree with the previous ones.

Note that we cannot use the above definition for $C=0$, in which the vector $\tilde{n}^{a}$ is not well defined. Before closing this section, let us see when such a case occurs. Equation (4.20) can be rewritten as

$$
C^{2}=\left(\Phi_{1}^{\dagger} \Phi_{1}\right)^{2}+\left(\Phi_{2}^{\dagger} \Phi_{2}\right)^{2}+2\left(\Phi_{1}^{\dagger} \sigma^{a} \Phi_{1}\right)\left(\Phi_{2}^{\dagger} \sigma^{a} \Phi_{2}\right) .
$$

Using the Fierz identity (A3), we have

$2\left(\Phi_{1}^{\dagger} \sigma^{a} \Phi_{1}\right)\left(\Phi_{2}^{\dagger} \sigma^{a} \Phi_{2}\right)=4\left|\Phi_{1}^{\dagger} \Phi_{2}\right|^{2}-2\left|\Phi_{1}\right|^{2}\left|\Phi_{2}\right|^{2}$,

and thus

$$
C^{2}=\left(\left|\Phi_{1}\right|^{2}-\left|\Phi_{2}\right|^{2}\right)^{2}+4\left|\Phi_{1}^{\dagger} \Phi_{2}\right|^{2} \geq 0 .
$$

The equality holds when

$$
\left|\Phi_{1}\right|^{2}=\left|\Phi_{2}\right|^{2}, \quad \Phi_{1}^{\dagger} \Phi_{2}=0
$$

in which the second term in Eq. (4.18) vanishes, and thus all $U(1)$ subgroups in $S U(2)_{W}$ are equivalent and not mixed with the $U(1)_{Y}$ component.

\section{TOPOLOGICAL PROPERTIES OF THE NAMBU MONOPOLE IN THE 2HDM}

Here we show that the Nambu monopole in the 2HDM has a nontrivial topological structure as for the 't HooftPolyakov monopole and the Wu-Yang monopole, unlike in the SM. This is one of the main results of this paper. In the first subsection, we first give a brief review of the electroweak strings and Nambu monopole in the 2HDM. The Nambu monopole in the 2HDM is a magnetic monopole attached with two topological $Z$ strings on the opposite sides $[58,59]$. After that we look at the topological structure, especially the fiber bundle of the electromagnetic gauge field of the monopole. As we stated above, we assume the $U(1)_{a}$ and $\left(\mathbb{Z}_{2}\right)_{\mathrm{C}}$ symmetries (4.5), but they are not essential for the topological structure of the fiber bundle.

\section{A. Nambu monopole in the 2HDM}

We first consider vortex solutions in the 2HDM. As studied in Refs. [42,44,45,50,51], the 2HDM with the $U(1)_{a}$ symmetry [Eq. (4.5)] admits topological vortex solutions. They can have (approximate) non-Abelian moduli (it can be genuine non-Abelian moduli in the limit of $g^{\prime} \rightarrow 0$ ) and confine the electroweak magnetic fluxes inside them, and hence are called the electroweak strings.

One of them is called the $(1,0)$ string, whose field configuration is given by

$$
\begin{aligned}
H^{(1,0)} & =v\left(\begin{array}{cc}
f^{(1,0)}(\rho) e^{i \varphi} & 0 \\
0 & h^{(1,0)}(\rho)
\end{array}\right), \\
Z_{i}^{(1,0)} & =-\frac{\cos \theta_{\mathrm{W}} \frac{\epsilon_{3 i j} x^{j}}{g}\left(1-w^{(1,0)}(\rho)\right),}{\rho^{2}}
\end{aligned}
$$

where $\rho \equiv \sqrt{x^{2}+y^{2}}$ and $\varphi$ is the rotation angle around the $z$ axis. The functions $f^{(1,0)}, h^{(1,0)}$, and $w^{(1,0)}$ satisfy the boundary conditions

$$
\begin{array}{ll}
f^{(1,0)}(\infty)=h^{(1,0)}(\infty)=1, & w^{(1,0)}(\infty)=0, \\
f^{(1,0)}(0)=\partial_{\rho} h^{(1,0)}(0)=0, & w^{(1,0)}(0)=1,
\end{array}
$$

so that the configurations are regular at $\rho=0$. The precise forms of the functions are determined by solving the EoMs, but they are irrelevant in our following argument. In Eq. (5.2) we have used the definition of the $Z$ gauge field [Eq. (4.11)], but it becomes trivial since the unit vector $n^{a}$ is constant $n^{a}=(0,0,-1)$ in the vortex configuration (5.1).

Due to the $\left(\mathbb{Z}_{2}\right)_{\mathrm{C}}$ symmetry in Eq. (4.5), we have another stable vortex solution called the $(0,1)$ string:

$$
\begin{aligned}
& H^{(0,1)}=v\left(\begin{array}{cc}
h^{(0,1)}(\rho) & 0 \\
0 & f^{(0,1)}(\rho) e^{i \varphi}
\end{array}\right), \\
& Z_{i}^{(0,1)}=\frac{\cos \theta_{\mathrm{W}}}{g} \frac{\epsilon_{3 i j} x^{j}}{\rho^{2}}\left(1-w^{(0,1)}(\rho)\right),
\end{aligned}
$$


where the profile functions $f^{(0,1)}, h^{(0,1)}$, and $w^{(0,1)}$ also satisfy the boundary conditions (5.3) and (5.4). The tension of the $(0,1)$ string is degenerate with that of the $(1,0)$ string as a result of the $\left(\mathbb{Z}_{2}\right)_{\mathrm{C}}$ symmetry. ${ }^{8}$ These configurations are not in the vacuum around $\rho=0$, i.e., the cores of the two $Z$ strings. Note that, in contrast to the case of the SM, the electroweak symmetry is not restored even around the string cores $\rho \sim 0$ because one doublet vanishes on $\rho \sim 0$ while the other does not [for instance, $f^{(1,0)}(0)=0$ but $\left.h^{(1,0)}(0) \neq 0\right]$. Therefore, one can even define the electromagnetic field inside the strings. This fact plays a crucial role for the topological structure of the Nambu monopole in the 2HDM, as seen below.

By rewriting Eqs. (5.1) and (5.5) as

$$
\begin{gathered}
H^{(1,0)}=v e^{i \frac{\varphi}{2}} e^{i \frac{\varphi}{2} \sigma_{3}}\left(\begin{array}{cc}
f^{(1,0)} & 0 \\
0 & h^{(1,0)}
\end{array}\right), \\
H^{(0,1)}=v e^{i \frac{\varphi}{2}} e^{-i \frac{\varphi}{2} \sigma_{3}}\left(\begin{array}{cc}
h^{(0,1)} & 0 \\
0 & f^{(0,1)}
\end{array}\right),
\end{gathered}
$$

it is clear that both the $(1,0)$ and $(0,1)$ strings have winding number $1 / 2$ for the global $U(1)_{a}$ symmetry, and thus they are topological vortex strings of the global type. Similarly to standard global vortices, their tensions (masses per unit length) logarithmically diverge.

On the other hand, they also have winding number $\pm 1 / 2$ inside the gauge orbit $U(1)_{Z} \in S U(2)_{W} \times U(1)_{Y}$, which leads to the $Z$ fluxes flowing inside them. The magnitudes of the fluxes of $(1,0)$ and $(0,1)$ strings are

$$
\Phi_{Z}^{(1,0)}=\frac{2 \pi \cos \theta_{\mathrm{W}}}{g}, \quad \Phi_{Z}^{(0,1)}=-\frac{2 \pi \cos \theta_{\mathrm{W}}}{g}
$$

along the $z$ axis, respectively. They are half of that of a nontopological $Z$ string in the SM because of the half winding number. The $Z$ flux is squeezed into a flux tube. In other words, contributions to the energy from the nonAbelian parts do not diverge.

There are physically different string configurations with a different (non-Abelian) magnetic flux, with a common winding number $1 / 2$ for the global $U(1)_{a}$. Since they belong to the same topological sector classified by the first homotopy group $\pi_{1}\left(U(1)_{a}\right)$, one can continuously deform one into the other (with some energy cost). ${ }^{9}$ Among them, the above two $Z$ strings are the lightest configurations as long as $m_{2}=\alpha_{4}=0$ and $\alpha_{3} \leq 0$ in the potential (4.4) [44,59], and thus they are energetically stable.

\footnotetext{
${ }^{8}$ One can check that they are related by the $\left(\mathbb{Z}_{2}\right)_{\mathrm{C}}$ transformation (4.5).

${ }^{9}$ In a certain limit of $e=0$ with the custodial symmetry, they can be continuously deformed into each other without energy cost by changing moduli.
}

A configuration describing the Nambu monopole is obtained by continuously connecting the two $Z$ strings with the $U(1)_{a}$ topological winding number kept, in which a junction point is a source for the $Z$ and electromagnetic fluxes, and hence is a magnetic monopole. We consider the case that $(1,0)$ and $(0,1)$ strings are put on the positive and negative side of the $z$ axis $(\theta=0, \pi)$, respectively, and the monopole (junction point) is located at the origin. At large distances from the origin, i.e., $r \rightarrow \infty$, the Higgs field $H$ and the gauge fields $W_{\mu}$ and $Y_{\mu}$ describing the monopole (with the strings) are given as

$$
\begin{array}{r}
H=v\left(\begin{array}{cc}
h_{1}(\theta) \sin \frac{\theta}{2} & h_{2}(\theta) \cos \frac{\theta}{2} \\
-e^{i \varphi} f_{1}(\theta) \cos \frac{\theta}{2} & e^{i \varphi} f_{2}(\theta) \sin \frac{\theta}{2},
\end{array}\right) \\
g W_{i}^{a}=-\cos ^{2} \theta_{W} n^{a} j(\theta) \cos \theta \partial_{i} \varphi-\epsilon^{a b c} n^{b} \partial_{i} n^{c}, \\
g^{\prime} Y_{i}=-\sin ^{2} \theta_{W} k(\theta) \cos \theta \partial_{i} \varphi .
\end{array}
$$

The profile functions $f_{1}, h_{1}$ and $f_{2}, h_{2}$ satisfy similar boundary conditions as those of the $(1,0)$ and $(0,1)$ strings at the north pole $(\theta=0)$ and south pole $(\theta=\pi)$, respectively,

$$
\begin{array}{ll}
f_{1}(0)=0, & \partial_{\theta} h_{1}(0)=0, \\
f_{2}(\pi)=0, & \partial_{\theta} h_{2}(\pi)=0,
\end{array}
$$

and they rapidly approach unity as we move from the north and south pole, respectively. In addition, the profile functions $j$ and $k$ satisfy the conditions,

$$
j(0)=j(\pi)=k(0)=k(\theta)=0
$$

and approach to unity far away from the poles. Equation (5.10) indeed describes the $(1,0)$ string $[(0,1)$ string] at $\theta \sim 0(\theta \sim \pi)$ up to the $S U(2)_{W}$ gauge transformation. ${ }^{10}$ Thanks to the boundary conditions, the configuration does not have line singularities at $\theta=0, \pi$. Note that one needs to introduce further $r$-dependent profile functions in order to smear the point-like singularity at $r=0$ and obtain a true solution of the EoMs. Nevertheless, we do not care about the singularity at $r=0$ because we are interested in topological properties of the monopole at large distances from the monopole $r \rightarrow \infty$.

It is worthwhile to look at the asymptotic forms of Eqs. (5.10)-(5.12) far away from the north and south poles. This is equivalent to considering an infinitely thin

\footnotetext{
${ }^{10}$ This is clear when one applies the $S U(2)_{W}$ gauge transformation $H \rightarrow U H$ on $U$, which satisfies

$$
\left.U\right|_{\theta=\pi}=1_{2 \times 2},\left.\quad U\right|_{\theta=0}=-i \sigma_{2}
$$
}


monopole and strings. Using the asymptotic behaviors in Eqs. (5.13)-(5.15), we have

$$
\begin{gathered}
H=v\left(\begin{array}{cc}
\sin \frac{\theta}{2} & \cos \frac{\theta}{2} \\
-e^{i \varphi} \cos \frac{\theta}{2} & e^{i \varphi} \sin \frac{\theta}{2}
\end{array}\right), \\
g W_{i}^{a}=-\cos ^{2} \theta_{W} n^{a} \cos \theta \partial_{i} \varphi-\epsilon^{a b c} n^{b} \partial_{i} n^{c}, \\
g^{\prime} Y_{i}=-\sin ^{2} \theta_{W} \cos \theta \partial_{i} \varphi .
\end{gathered}
$$

This asymptotic configuration seems to have line singularities at $\theta=0$ and $\pi$, which correspond to the cores of the two $Z$ strings $[(1,0)$ and $(0,1)$ strings, respectively] as artifacts of asymptotic forms, and is valid only at large distances from the line singularities, $\rho \rightarrow \infty$. [Indeed, the singularities are avoided because $f_{1}(0)=0$ and $f_{2}(\pi)=0$ in Eq. (5.10).]

Let us look at the $Z$ and electromagnetic fluxes around the monopole. To do so, we first consider the vectors $n_{1}^{a}$ and $n_{2}^{a}$ defined by Eq. (4.6). From Eq. (5.10), we have

$$
\begin{aligned}
& \Phi_{1}^{\dagger} \sigma^{a} \Phi_{1}=v^{2}\left(f_{1} \frac{x}{r}, f_{1} \frac{y}{r}, f_{1}^{2} c_{1 / 2}^{2}-h_{1}^{2} s_{1 / 2}^{2}\right), \\
& \Phi_{2}^{\dagger} \sigma^{a} \Phi_{2}=v^{2}\left(f_{2} \frac{x}{r}, f_{2} \frac{y}{r}, h_{2}^{2} c_{1 / 2}^{2}-f_{2}^{2} s_{1 / 2}^{2}\right),
\end{aligned}
$$

with $c_{1 / 2} \equiv \cos \frac{\theta}{2}$ and $s_{1 / 2} \equiv \sin \frac{\theta}{2}$, and the unit vectors defined by Eq. (4.6) are

$$
\begin{aligned}
& n_{1}^{a}=\frac{f_{1} h_{1}}{f_{1}^{2} c_{1 / 2}^{2}+h_{1}^{2} s_{1 / 2}^{2}}\left(\frac{x}{r}, \frac{y}{r}, \frac{f_{1}^{2} c_{1 / 2}^{2}-h_{1}^{2} s_{1 / 2}^{2}}{f_{1} h_{1}}\right), \\
& n_{2}^{a}=\frac{f_{2} h_{2}}{h_{2}^{2} c_{1 / 2}^{2}+f_{2}^{2} s_{1 / 2}^{2}}\left(\frac{x}{r}, \frac{y}{r}, \frac{h_{2}^{2} c_{1 / 2}^{2}-f_{2}^{2} s_{1 / 2}^{2}}{f_{2} h_{2}}\right) .
\end{aligned}
$$

Note that $n_{1}^{a}$ and $n_{2}^{a}$ are not well defined at $\theta=0$ and $\theta=\pi$, respectively, because the denominators vanish.

Furthermore, we require that the $U(1)_{\mathrm{EM}}$ is not broken everywhere, which leads to $n_{1}^{a}=n_{2}^{a}$ everywhere except for $\theta=0, \pi{ }^{11}$ We can explicitly confirm that this is true by constructing a full solution of the EoMs, as in Refs. [58,59]. This leads to two conditions for the profile functions $f_{i}$ and $h_{i}$. The expressions are rather complicated so we do not present them, although they are assumed implicitly in the following.

We next consider the unit vector $n^{a}$ defined by Eq. (4.9). Substituting Eqs. (5.21) and (5.22), we obtain

\footnotetext{
${ }^{11}$ Note that although $n_{1}^{a}\left(n_{2}^{a}\right)$ is not defined at $\theta=0(\theta=\pi)$, the $U(1)_{\mathrm{EM}}$ is not broken even at $\theta=0, \pi$. It is broken only when both $n_{1}^{a}$ and $n_{2}^{a}$ are well defined and $n_{1}^{a} \neq n_{2}^{a}$.
}

$$
\begin{aligned}
n^{a}= & \frac{f_{1} h_{1}+f_{2} h_{2}}{\left(f_{1}^{2}+h_{2}^{2}\right) c_{1 / 2}^{2}+\left(f_{2}^{2}+h_{1}^{2}\right) s_{1 / 2}^{2}} \\
& \times\left(\frac{x}{r}, \frac{y}{r}, \frac{\left(h_{2}^{2}+f_{1}^{2}\right) c_{1 / 2}^{2}-\left(h_{1}^{2}+f_{2}^{2}\right) s_{1 / 2}^{2}}{f_{1} h_{1}+f_{2} h_{2}}\right),
\end{aligned}
$$

which is regular and well defined everywhere, even at $\theta=0, \pi$. Indeed, $n^{a}=n_{2}^{a}=(0,0,1)$ for $\theta=0$ and $n^{a}=$ $n_{1}^{a}=(0,0,-1)$ for $\theta=\pi$. (Recall that $f_{1} \rightarrow 0$ and $f_{2} \rightarrow 0$ for $\theta \rightarrow 0$ and $\pi$, respectively.) As we move from the string core, $\rho \rightarrow \infty, n^{a}$ approaches the hedgehog one as in the 't Hooft-Polyakov case,

$$
n^{a}=(\sin \theta \cos \varphi, \sin \theta \sin \varphi, \cos \theta)^{t}=\frac{x^{a}}{r} .
$$

On the other hand, it becomes slightly deformes around $\theta \sim 0, \pi$, as depicted in Fig. 2.

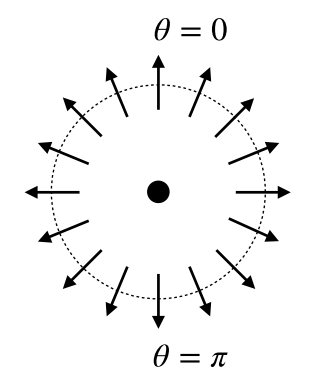

(a) 't Hooft-Polyakov monopole

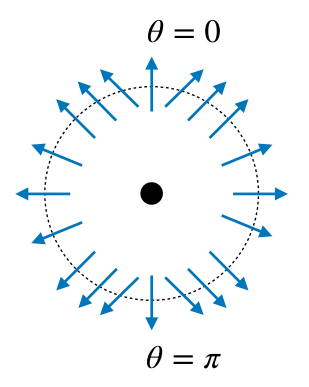

(b) Nambu monopole in $2 \mathrm{HDM}$
FIG. 2. Structure of the unit vectors $n^{a}$. (a): for the 't HooftPolyakov monopole, $n^{a}$ is defined by Eq. (2.5) and is the hedgehog structure. (b): for the Nambu monopole in the 2HDM, $n^{a}$ is given by Eq. (5.25) and is not the hedgehog structure. It becomes deformed around $\theta \sim 0$ and $\pi$.
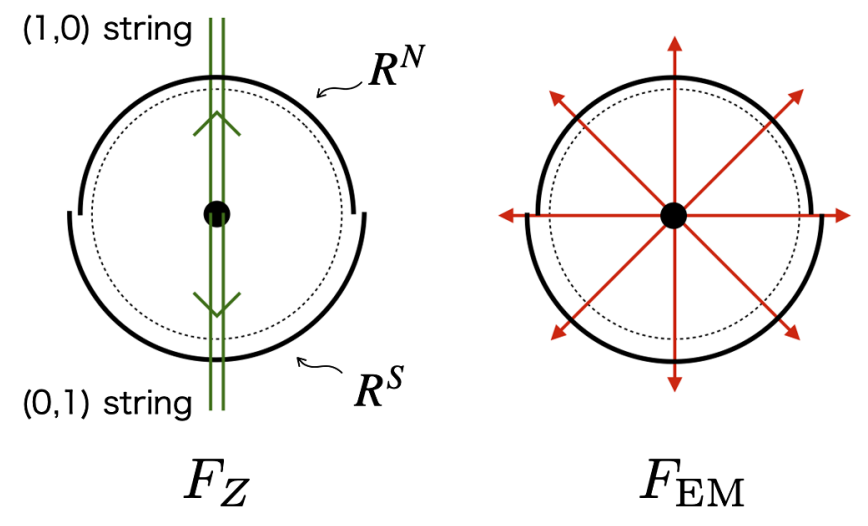

FIG. 3. Schematic picture of the two patches $R^{N}$ and $R^{S}$ surrounding the Nambu monopole. The $(1,0)$ and $(0,1)$ strings pass through $R^{N}$ and $R^{S}$, respectively. The electromagnetic flux $\Phi_{\mathrm{EM}}=4 \pi \sin \theta_{\mathrm{W}} / g$ spreads radially from the monopole (right panel), while the $Z$ flux, $\Phi_{Z}=2 \pi \cos \theta_{\mathrm{W}} / g$, is confined to each $Z$ string $[(1,0)$ or $(0,1)$ string] (left panel). 
For later use, we rewrite $n^{a}$ at finite $\rho$ [Eq. (5.23)] as

$$
n^{a}=(\sin \Theta \cos \varphi, \sin \Theta \sin \varphi, \cos \Theta),
$$

where $\Theta(\theta)$ is a "deformed zenith angle," defined by

$$
\begin{gathered}
\cos \Theta \equiv \frac{\left(h_{2}^{2}+f_{1}^{2}\right) c_{1 / 2}^{2}-\left(h_{1}^{2}+f_{2}^{2}\right) s_{1 / 2}^{2}}{\left(f_{1}^{2}+h_{2}^{2}\right) c_{1 / 2}^{2}+\left(f_{2}^{2}+h_{1}^{2}\right) s_{1 / 2}^{2}}, \\
\sin \Theta \equiv \frac{\left(f_{1} h_{1}+f_{2} h_{2}\right)}{\left(f_{1}^{2}+h_{2}^{2}\right) c_{1 / 2}^{2}+\left(f_{2}^{2}+h_{1}^{2}\right) s_{1 / 2}^{2}} \frac{\sqrt{x^{2}+y^{2}}}{r} .
\end{gathered}
$$

Note that $\cos ^{2} \Theta+\sin ^{2} \Theta=1$ since $\left|n^{a}\right|^{2}=1$, and $\Theta(0)=0, \Theta(\pi)=\pi$. In the matrix representation,

$$
n \equiv n^{a} \frac{\sigma^{a}}{2}=\frac{1}{2}\left(\begin{array}{cc}
\cos \Theta & \sin \Theta e^{-i \varphi} \\
\sin \Theta e^{i \varphi} & -\cos \Theta
\end{array}\right) .
$$

Because $n_{1}^{a}=n_{2}^{a}$ holds everywhere except for $\theta=0, \pi$, we can define the field strengths of the electromagnetic and the $Z$ gauge bosons by using Eqs. (4.14) and (4.15) and the vector $n^{a}$ [Eq. (5.25)]. However, the expressions are rather complicated, and hence here we only consider the asymptotic forms far from the string cores $\rho \rightarrow \infty$ by substituting the asymptotic forms (5.16) [or Eq. (5.24)] into Eqs. (4.14) and (4.15). Then, we obtain [58]

$$
\begin{gathered}
F_{i j}^{Z}=\frac{2 \pi \cos \theta_{\mathrm{W}}}{g} \frac{z}{|z|} \epsilon_{3 i j} \delta(x) \delta(y), \\
F_{i j}^{\mathrm{EM}}=\frac{\sin \theta_{\mathrm{W}}}{g} \epsilon^{a i j} \frac{x^{a}}{r^{3}} .
\end{gathered}
$$

From Eq. (5.30), it is clear that there is a magnetic flux emanating from the origin in a spherical hedgehog form. The total amount of magnetic flux $\Phi_{\mathrm{EM}}$ can be calculated by integrating the flux density $B_{i} \equiv \frac{1}{2} \epsilon_{i j k} F_{j k}^{\mathrm{EM}}$ as

$$
\Phi_{\mathrm{EM}}=\int d^{3} x \partial_{i} B_{i}=\frac{4 \pi \sin \theta_{\mathrm{W}}}{g} .
$$

In addition, from Eq. (5.29) the $Z$ fluxes only exist on the $z$ axis as

$$
\begin{gathered}
\left.\Phi_{Z}\right|_{z>0}=\left.\int d x^{2} F_{i j}^{Z}\right|_{z>0}=\frac{2 \pi \cos \theta_{\mathrm{W}}}{g}=\Phi_{Z}^{(1,0)}, \\
\left.\Phi_{Z}\right|_{z<0}=\left.\int d x^{2} F_{i j}^{Z}\right|_{z<0}=-\frac{2 \pi \cos \theta_{\mathrm{W}}}{g}=\Phi_{Z}^{(0,1)},
\end{gathered}
$$

flowing on the positive and negative sides of the $z$ axis, respectively, from the origin. These $Z$-flux magnitudes agree with those of the $Z$ strings in Eq. (5.9). The total $Z$-flux magnitude flowing from the monopole at the origin can be calculated as
$\Phi_{Z}=\int d x^{3} \partial_{i} B_{i}^{Z}=\left.\Phi_{Z}\right|_{z>0}-\left.\Phi_{Z}\right|_{z<0}=\frac{4 \pi \cos \theta_{\mathrm{W}}}{g}$,

with $B_{i}^{Z} \equiv \frac{1}{2} \epsilon_{i j k} F_{j k}^{Z}$. Therefore, the total $Z$ flux agrees with that of the Nambu monopole in the SM [Eq. (3.20)]. See Fig. 3 for a schematic picture of the $Z$ and electromagnetic fluxes from the monopole.

It is worth demonstrating the topological current of $U(1)_{a}$ in the configuration. The topological current, corresponding to the winding of the $U(1)_{a}$ phase of the Higgs field, is defined by

$$
\begin{gathered}
\mathcal{A}_{i} \equiv \epsilon_{i j k} \partial_{j} \mathcal{J}_{k}, \\
\mathcal{J}_{i} \equiv-i \operatorname{tr}\left[H^{\dagger} D_{i} H-\left(D_{i} H\right)^{\dagger} H\right] .
\end{gathered}
$$

Importantly, $\mathcal{A}_{i}$ is topologically conserved, $\partial_{i} \mathcal{A}_{i}=0$, and is independent of $z$, as can be seen by substituting Eq. (5.16). This indicates that not only the string parts but also the monopole itself at the origin have the topological charge of $U(1)_{a}$.

\section{B. Fiber bundle for the Nambu monopole}

Here we discuss the topological structure of the electromagnetic field of the Nambu monopole. In particular, we investigate a fiber bundle consisting of the base space $S^{2}$ surrounding the monopole and the fiber $U(1)_{\mathrm{EM}}$.

Importantly, since the strings pass through the base space $S^{2}$ at $\theta=0, \pi$ (see Fig. 3), we cannot use the asymptotic forms (5.16)-(5.18) on the sphere $S^{2}$, which are singular at the north and south poles. Thus, we have to use the smeared one [Eqs. (5.10)-(5.18)] without the line singularities.

We should stress that the unit vector $n^{a}$ [Eq. (5.25)] is homotopically equivalent to the hedgehog one [Eq. (5.24)], i.e., the map

$$
\begin{aligned}
n^{a}(\theta, \varphi): S^{2} & =\{0 \leq \theta \leq \pi, 0 \leq \varphi<2 \pi\} \mapsto \\
S^{2} & =\left\{n^{a}=\left(n^{1}, n^{2}, n^{3}\right) \mid\left(n^{1}\right)^{2}+\left(n^{2}\right)^{2}+\left(n^{3}\right)^{2}=1\right\}
\end{aligned}
$$

has a winding number of unity. The target space $S^{2}$ corresponds to the order parameter space $S U(2)_{W} / U(1)_{n}$, where $U(1)_{n}$ is a subgroup of $S U(2)_{W}$ that leaves $n^{a}$ invariant. ${ }^{12}$ Therefore, $n^{a}$ has the same topological structure as the 't Hooft-Polyakov monopole [see Eq. (2.7) and surrounding text], and the Nambu monopole in the 2HDM can be regarded as an embedding of the 't Hooft-Polyakov monopole into the $S U(2)_{W}$ sector accompanied by the $U(1)_{Y}$ flux.

\footnotetext{
${ }^{12}$ Although $U(1)_{n}$ keeps $n^{a}$ invariant, it does not keep $\Phi_{1}$ or $\Phi_{2}$ invariant, and thus $U(1)_{n}$ itself is not an unbroken subgroup.
} 
To see this more clearly, let us first consider the fiber bundle of the $U(1)_{n}$ subgroup. Thanks to the fact that the unit vector $n^{a}$ in Eq. (5.23) or Eq. (5.25) is regular everywhere, we can define the $U(1)_{n}$ subgroup on the whole sphere $S^{2}$ surrounding the monopole, unlike in the case of the Nambu monopole in the SM. Thus, we should divide $S^{2}$ into two hemispheres $R^{N}$ and $R^{S}$ as in the case of the 't Hooft-Polyakov monopole (Sec. II),

$$
R^{N}: 0 \leq \theta \leq \frac{\pi}{2}, \quad R^{S}: \frac{\pi}{2} \leq \theta \leq \pi,
$$

which have an overlap region on the equator $\theta=\pi / 2$. What is different from the 't Hooft-Polyakov monopole is that we now have the $Z$ strings at $\theta=0$ and $\theta=\pi$, and thus the strings pass thro ugh $R^{N}$ and $R^{S}$ (see Fig. 3). However, this is irrelevant because $n^{a}$ has no singularity even at the centers of the $Z$ strings.

Then, we introduce the $S U(2)_{W}$ gauge transformations $U^{N}$ and $U^{S}$ defined on each hemisphere,

$$
\begin{aligned}
R^{N}: U^{N}(\theta, \varphi) & =e^{-i \sigma^{3} \varphi / 2} e^{-i \sigma^{2} \Theta / 2} e^{i \sigma^{3} \varphi / 2} \\
& =\left(\begin{array}{cc}
\cos \frac{\Theta}{2} & -\sin \frac{\Theta}{2} e^{-i \varphi} \\
\sin \frac{\Theta}{2} e^{i \varphi} & \cos \frac{\Theta}{2}
\end{array}\right), \\
R^{S}: U^{S}(\theta, \varphi) & =e^{-i \sigma^{3} \varphi / 2} e^{-i \sigma^{2}(\pi-\Theta) / 2} e^{i \sigma^{3} \varphi / 2}\left(-i \sigma^{2}\right) \\
& =\left(\begin{array}{cc}
e^{-i \varphi} \cos \frac{\Theta}{2} & -\sin \frac{\Theta}{2} \\
\sin \frac{\Theta}{2} & e^{i \varphi} \cos \frac{\Theta}{2}
\end{array}\right)
\end{aligned}
$$

Note that we have used $\Theta$ instead of $\theta$. Both transformations bring the unit vector $n^{a}$ into the uniform vector in each region:

$$
\begin{aligned}
& U^{N}: n^{a} \frac{\sigma^{a}}{2} \rightarrow\left(U^{N}\right)^{\dagger} n^{a} \frac{\sigma^{a}}{2} U^{N}=\frac{\sigma^{3}}{2}, \\
& U^{S}: n^{a} \frac{\sigma^{a}}{2} \rightarrow\left(U^{S}\right)^{\dagger} n^{a} \frac{\sigma^{a}}{2} U^{S}=\frac{\sigma^{3}}{2} .
\end{aligned}
$$

Note that $U^{N}$ and $U^{S}$ are regular on each hemisphere $R^{N}$ and $R^{S}$. In this gauge, the subgroup $U(1)_{n}$ is uniformly defined as the $\sigma^{3}$ subgroup of $S U(2)_{W}$ on the two hemispheres.

Similarly to Sec. II, it follows from the single valuedness of $n^{a}$ at $\theta=\pi / 2$ that the transition function $\left(U^{S}\right)^{-1} U^{N}$ must leave $n^{a}$ invariant, i.e., $\left(U^{S}\right)^{-1} U^{N} \in U(1)_{n}$ [not $\left.U(1)_{\mathrm{EM}}\right]$. Indeed, noting that $\Theta=\pi / 2$ for $\theta=\pi / 2$, we have

$$
\left(U^{S}\right)^{-1} U^{N}=e^{i \varphi \sigma^{3}}
$$

which is the same as Eq. (2.31) and has a winding number of unity.
Thus, the first Chern number associated with $U(1)_{n}$ is obtained as

$$
\int_{S^{2}} c_{1}^{(n)}=\frac{g}{2 \pi} \int_{S^{2}} F^{n}=1
$$

with $F_{i j}^{n} \equiv n^{a} W_{i j}^{a}\left(\right.$ not $F_{i j}^{Z}$ or $\left.F_{i j}^{\mathrm{EM}}\right)$. Therefore, the topological structure of $U(1)_{n}\left(\subset S U(2)_{W}\right)$ is completely the same as the unbroken subgroup of the 't Hooft-Polyakov monopole.

Let us turn to the topological structure of $U(1)_{\mathrm{EM}}$, which is defined by the linear combination of $U(1)_{n}$ and $U(1)_{Y}$ [Eq. (4.15)]. Since the divergence of the $U(1)_{Y}$ flux is always zero, the global structure of the fiber $U(1)_{Y}$ is trivial, i.e., the fiber bundle is a direct product: $S^{2} \times U(1)_{Y}$. Therefore, we can concentrate on the $U(1)_{n}$ part. We consider the first Chern number associated with $U(1)_{\mathrm{EM}}$, which is given by ${ }^{13}$

$$
\int_{S^{2}} c_{1}^{(\mathrm{EM})} \equiv \frac{-g}{2 \pi \sin \theta_{W}} \int_{S^{2}} F^{\mathrm{EM}}=\frac{g}{2 \pi} \int_{S^{2}} F^{n}=\int_{S^{2}} c_{1}^{(n)}=1 .
$$

This means that the magnetic charge is quantized because $c_{1}^{(n)}$ is quantized as Eq. (5.44). Such a quantization originates from the fact that the topological structure of $U(1)_{n}$ of this monopole has the same topology as that of the 't Hooft-Polyakov monopole (or the Wu-Yang monopole bundle).

Finally, let us consider the Dirac quantization condition. When a test particle with $U(1)_{Y}$ hypercharge $Y$ and weak isospin $T_{3}=1 / 2$ goes around the equator $\theta=\pi / 2$, it receives an $\mathrm{AB}$ phase, given by

$$
\theta_{\mathrm{AB}}=e Q \oint_{\theta=\pi / 2} d x_{i} A_{i}+g_{Z} T_{Z} \oint_{\theta=\pi / 2} d x_{i} Z_{i}
$$

where we have used $e=g \sin \theta_{W}$ and

$$
\begin{gathered}
Q \equiv T_{3}+\frac{Y}{2}, \\
T_{Z} \equiv T_{3}-\sin ^{2} \theta_{W} Q .
\end{gathered}
$$

Note that in Eq. (5.46) there is also a contribution from the $Z$ flux confined inside the two $Z$ strings (the second term), in addition to that from the electromagnetic gauge field (the first term). Using the definitions of the gauge fields in Eqs. (4.11) and (4.13) and noting that $n^{a}=(0,0,1)$ on each patch, we obtain

\footnotetext{
${ }^{13}$ We choose a normalization factor for $c_{1}^{(\mathrm{EM})}$ such that it takes an integer value under the integration.
} 


$$
\theta_{\mathrm{AB}}=\oint_{\theta=\pi / 2} d x_{i}\left(-g T_{3} W_{i}^{3}+g^{\prime} \frac{Y}{2} Y_{i}\right)
$$

The second term in Eq. (5.49) vanishes because the $U(1)_{Y}$ fiber is trivial. We can calculate the $\mathrm{AB}$ phases in the two patches $R^{N}$ and $R^{S}$. Noting that the difference of $W_{i}^{3}$ on the two patches is given by

$$
\Delta W_{i}^{3}=\frac{-i}{g} \operatorname{Tr}\left(\sigma_{3}\left[\left(U^{S}\right)^{-1} U^{N}\right]^{\dagger} \partial_{i}\left(U^{S}\right)^{-1} U^{N}\right),
$$

the condition for the single valuedness of the wave function of the particle requires that the difference between the two patches,

$$
\Delta \theta_{A B}=g T_{3} \oint_{\theta=\pi / 2} d x_{i} \Delta W_{i}^{3}=4 \pi T_{3}
$$

must be an integer multiple of $2 \pi$,

$$
4 \pi T_{3}=2 \pi n(n \in \mathbb{Z}) \Leftrightarrow T_{3}=\frac{n}{2},
$$

which is the Dirac quantization condition derived from the Nambu monopole in the 2HDM. Recalling that $T_{3}=1 / 2$, this is automatically satisfied with $n=1$. Remarkably, the Dirac quantization condition for the Nambu monopole in the 2HDM does not ensure the quantization of the electric charges because the fiber bundle of $U(1)_{Y}$ is trivial and the $U(1)_{Y}$ hypercharge cannot be quantized by the monopole.

Before closing this section, we give some remarks. In the $\mathrm{SM}$, in the presence of the Nambu monopole, the magnetic charge is not quantized and the $U(1)_{\mathrm{EM}}$ and $Z$ gauge fields cannot have any topologically nontrivial structures. This is because the electroweak symmetry is restored and hence $U(1)_{\mathrm{EM}}$ cannot be defined at the center of the $Z$ string. On the other hand, in the 2HDM the magnetic charge is quantized as in Eq. (5.45) because the topological charge (first Chern number) $c_{1}^{(n)}$ is quantized. This originates from the fact that the Nambu monopole in the 2HDM is an embedding of the 't Hooft-Polyakov monopole into the $S U(2)_{W}$ sector. What makes the embedding possible is that $n^{a}$ defined by Eq. (4.9) is regular and well defined everywhere on the infinitely large sphere $S^{2}$ surrounding the monopole. The regularity means that the electroweak symmetry is not restored on the whole sphere, even on the cores of the $Z$ strings. In this sense, the Nambu monopole in the $2 \mathrm{HDM}$ is indeed a true magnetic monopole although it is attached with the strings. This topological argument is still valid even when the $U(1)_{a}$ and $\left(\mathbb{Z}_{2}\right)_{\mathrm{C}}$ symmetries are explicitly broken in the potential because the breaking effects do not affect the topology of the 't Hooft-Polyakov embedding in $S U(2)_{W} \rightarrow U(1)_{n}$. However, stability is no longer ensured in the presence of such breaking terms because we have no nontrivial second homotopy group $\pi_{2}$ in the full symmetry breaking $S U(2)_{W} \times U(1)_{Y} \rightarrow U(1)_{\mathrm{EM}}$. The reader should not confuse the topological properties of this monopole with the topological stability. To stabilize the monopole, we need nontrivial $\pi_{0}$ and $\pi_{1}$ associated with the $\left(\mathbb{Z}_{2}\right)_{\mathrm{C}}$ and $U(1)_{a}$ symmetries, as studied in Ref. [58]. See Ref. [59] for the unstable case without $\left(\mathbb{Z}_{2}\right)_{\mathrm{C}}$.

\section{DYON IN THE 2HDM}

Similarly to the electroweak dyon in the SM studied in Ref. [15], we can consider a dyon configuration in the 2HDM. To do this, we consider the gauge moduli of the Nambu monopole described by Eqs. (5.16)-(5.18). Hereafter, we denote the Nambu monopole configuration in the $2 \mathrm{HDM}$ by $\bar{H}, \bar{W}_{\mu}^{a}$, and $\bar{Y}_{\mu}$. The $S U(2)_{W}$ adjoint vector $n^{a}$ defined in Eq. (4.9) can be rewritten in terms of the $2 \times 2$ matrix $H$ as

$$
n^{a}=\frac{\operatorname{tr}\left(\sigma^{3} H^{\dagger} \sigma^{a} H\right)}{\operatorname{tr}\left(H^{\dagger} H\right)}
$$

which satisfies

$$
\left(\bar{D}_{\mu} n\right)^{a}=0
$$

at large distances from the strings, $\rho \rightarrow \infty$, where $\bar{D}_{\mu}$ is the covariant derivative consisting of $\bar{W}_{\mu}$ and $\bar{Y}_{\mu}$. In addition, the following identity holds:

$$
\bar{H} \sigma^{3}+n^{a} \sigma^{a} \bar{H}=0 .
$$

This means that $\bar{H}$ is invariant under the $U(1)_{\mathrm{EM}}$ gauge transformation ${ }^{14}$ :

$$
\exp \left(-i \alpha n^{a} \frac{\sigma_{a}}{2}\right) \bar{H} \exp \left(-i \alpha \frac{\sigma_{3}}{2}\right)=\bar{H},
$$

with $\alpha$ being an arbitrary function.

Due to the $U(1)_{\mathrm{EM}}$ symmetry, the monopole has a modulus (zero mode) under the following transformation:

$$
\begin{array}{r}
\bar{H} \rightarrow \exp \left[-i \alpha n^{a} \frac{\sigma_{a}}{2}\right] \bar{H} \exp \left[-i \alpha \frac{\sigma_{3}}{2}\right](=\bar{H}), \\
g \bar{W}_{\mu} \rightarrow \exp \left[-i \alpha n^{a} \frac{\sigma_{a}}{2}\right]\left(g \bar{W}_{\mu}-i \partial_{\mu}\right) \exp \left[i \alpha n^{a} \frac{\sigma_{a}}{2}\right], \\
g^{\prime} \bar{Y}_{\mu} \rightarrow g^{\prime} \bar{Y}_{\mu}-\exp [i \alpha] i \partial_{\mu} \exp [-i \alpha]\left(=g^{\prime} \bar{Y}_{\mu}\right),
\end{array}
$$

for constant $\alpha \in[0,2 \pi)$.

Let us make a dyon configuration from the Nambu monopole. In the following, we are only interested in the

${ }^{14}$ The $U(1)_{\mathrm{EM}}$ transformation is defined as a combination of the $U(1)_{n}$ and $U(1)_{Y}$ transformations. 
asymptotic forms at large distances from the string cores $\rho \rightarrow \infty$. If one wants to obtain a true configuration, it is necessary to solve the full EoMs. The asymptotic form of the dyon configuration is obtained by giving the monopole a time-dependent excitation using a function $\gamma(x)=\gamma(t, \boldsymbol{x})$,

$$
\begin{gathered}
H=\bar{H}, \\
g W_{\mu}^{a}=g \bar{W}_{\mu}^{a}-\delta_{\mu 0}\left(\bar{D}_{\mu}(n \gamma(x))\right)^{a}=g \bar{W}_{\mu}^{a}-\delta_{\mu 0} n^{a} \dot{\gamma}, \\
g^{\prime} Y_{\mu}=g^{\prime} \bar{Y}_{\mu}+\delta_{\mu 0} \partial_{\mu} \gamma(x)=g^{\prime} \bar{Y}_{\mu}+\delta_{\mu 0} \dot{\gamma},
\end{gathered}
$$

where $\dot{\gamma}=\partial_{t} \gamma$ and we have used Eq. (6.2) in Eq. (6.9). This excitation is regarded as applying the $U(1)_{\mathrm{EM}}$ transformation (6.5)-(6.7) only for the time components with $\alpha \rightarrow \gamma(x)$. Due to this, the field strengths are changed from those in the Nambu monopole. The difference is given as

$$
\begin{gathered}
\delta W_{i 0}^{a}=-\frac{\left(\bar{D}_{i}(n \dot{\gamma})\right)^{a}}{g}=-\frac{n^{a} \partial_{i} \dot{\gamma}}{g}, \\
\delta Y_{i 0}=+\frac{\partial_{i} \dot{\gamma}}{g^{\prime}},
\end{gathered}
$$

and $\delta W_{i j}=\delta Y_{i j}=0$. For the electromagnetic and $Z$ field strengths,

$$
\delta F_{i 0}^{\mathrm{EM}}=\frac{\sqrt{g^{2}+g^{\prime 2}}}{g g^{\prime}} \partial_{i} \dot{\gamma}
$$

and the others do not change.

Let us obtain $\gamma$ by solving the EoMs at large distances from the string core, $\rho \rightarrow \infty$. For the ansatz in Eqs. (6.8)(6.10), the EoMs reduce to the ordinary Maxwell equations for the electric field,

$$
\partial_{0} F_{i 0}^{\mathrm{EM}}=0, \quad \partial_{i} F_{0 i}^{\mathrm{EM}}=0,
$$

which lead to

$$
\partial_{i} \partial_{i} \dot{\gamma}=\partial_{0} \partial_{i} \dot{\gamma}=0
$$

Note that the other components of the fields do not receive backreactions from $\gamma$ because the massive gauge components decay exponentially at large distances and the Higgs field does not couple to $\gamma$. Therefore, the other EoMs are satisfied independently of $\gamma .{ }^{15}$

From Eq. (6.15), we have

$$
\dot{\gamma}=c_{0}(t)-\frac{\Lambda}{4 \pi r},
$$

\footnotetext{
${ }^{15}$ Of course, they are nonlinearly coupled around the cores of the dyon and the string.
}

where $\Lambda$ is an arbitrary constant and $c_{0}(t)$ is an arbitrary function of $t$. By the gauge-fixing condition [see Eq. (B5) in Appendix B], we impose $\ddot{\gamma}=0$ and hence $\dot{c}_{0}(t)=0$. We thus obtain a general solution of $\gamma$ as

$$
\gamma=\left(c_{0}-\frac{\Lambda}{4 \pi r}\right) t+\text { const. }
$$

Substituting this into Eq. (6.13), we then get

$$
E_{i}=F_{0 i}^{\mathrm{EM}}=\Lambda \frac{\sqrt{g^{2}+g^{\prime 2}}}{4 \pi g g^{\prime}} \frac{x_{i}}{r^{3}}=\frac{\Lambda}{e} \frac{1}{4 \pi} \frac{x_{i}}{r^{3}} .
$$

Therefore, the configuration in Eqs. (6.8)-(6.10) with Eq. (6.17) indeed describes a dyon configuration with electric charge $q=\Lambda / e$.

So far, the electric charge $q$ is arbitrary and continuous. We finally discuss the charge quantization condition for the dyon. Let us consider a pair of dyons with magnetic and electric charges $\left(q_{M}, q_{1}\right)$ and $\left(q_{M}, q_{2}\right)$, with $q_{M}=$ $4 \pi \sin ^{2} \theta_{W} / e$. From the quantization condition for angular momentum in quantum mechanics, we can derive the condition (see Ref. [15]) as ${ }^{16}$

$$
q_{1}-q_{2}=n e, \quad n \in \mathbb{Z} .
$$

If we use this condition for a dyon $\left(q_{1}=q\right)$ and monopole $\left(q_{2}=0\right)$ pair, we obtain

$$
q=n e, \quad n \in \mathbb{Z},
$$

and thus $\Lambda$ in Eq. (6.17) must be quantized as $\Lambda=n e^{2}$. Therefore, the electric charge of the dyon must be a multiple of the minimal charge $e$.

\section{CONCLUSION AND DISCUSSION}

We have studied the topological properties of the Nambu monopole in the 2HDM. In the SM, the Nambu monopole has no topological structures because the $U(1)_{\mathrm{EM}}$ gauge field cannot be defined on the center of the $Z$ string, where the electroweak symmetry is restored. Thus, a $U(1)_{\mathrm{EM}}$ fiber bundle over a spatial surface $S^{2}$ surrounding the monopole is topologically trivial: $U(1)_{\mathrm{EM}} \times S^{2}$. This is different from the 't Hooft-Polyakov monopole which has the same structure as the Wu-Yang description of the Dirac monopole, for which $U(1)_{\mathrm{EM}}$ is Hopf fibered over $S^{2}$ with the total

\footnotetext{
${ }^{16}$ Note that the analysis of the angular momentum around a pair of dyons in Ref. [15] cannot be justified in the SM because there is a singular point in the $Z$ string where the electromagnetic field cannot be defined, as stated in Sec. III. However, we can apply the result to the case of the dyons in the 2HDM, in which the electromagnetic field is well defined everywhere except for the center of the monopole or dyon.
} 
space $S^{3}$. As a result of the trivial topology, the magnetic charge is not topologically quantized for the Nambu monopole in the SM. On the other hand, the monopole in the 2HDM has the same topological structure as the 't Hooft-Polyakov monopole thanks to the fact that $U(1)_{n}$ and hence $U(1)_{\mathrm{EM}}$ are well defined everywhere (except for the center of the monopole), implying that the electroweak gauge symmetry is broken everywhere including the string cores. Concentrating on the $S U(2)_{W} \rightarrow U(1)_{n}$ sector, the Nambu monopole can be regarded as an embedding of the 't Hooft-Polyakov monopole, and the fiber bundle of $U(1)_{n}$ on the base space $S^{2}$ surrounding the monopole is nontrivial, $S^{3}$, as in the 't Hooft-Polyakov and Wu-Yang cases. Consequently, the $U(1)_{\mathrm{EM}}$ gauge field also has a nontrivial topology on $S^{2}$ and the electromagnetic flux is fractionally quantized. We have shown that the Dirac quantization condition (5.52) always holds for test particles charged under the electroweak gauge symmetry.

We have also shown the dyon configuration in the 2HDM. A stationary time-dependent excitation makes the Nambu monopole a dyon, having both magnetic and electric charges. Thus, the dyon behaves as a source for the electric and magnetic fields and the $Z$ magnetic flux. Considering quantum effects, the electric charge of the dyon is quantized by the minimal electric charge $e=g \sin \theta_{W}$.

Before closing this paper, some discussions are addressed here. It is known that the $C P$-violating $\theta$ term $g^{2} \theta F \tilde{F} / 32 \pi^{2}$ in the Lagrangian changes the 't HooftPolyakov monopole into a dyon with a fractional electric charge $q=(n-\theta / 2 \pi) e$, which is called the Witten effect [66]. Let us discuss the Witten effect in the 2HDM. When the couplings between the gauge fields and the SM fermions are neglected, the $\theta$ term of the $S U(2)_{W}$ gauge sector provides the same effect on the Nambu monopole in the 2 HDM. ${ }^{17}$ The couplings to the SM fermions make the $\theta$ term unphysical since it can be eliminated by a redefinition of the fermions and Higgs doublets. However, if the theory has any explicit $C P$-violating parameters, they provide similar effects through higher-order loop corrections after integrating out perturbations around the monopole configuration [66,67], resulting in the Nambu monopole with a fractional electric charge. In fact, the 2HDM with fermions in general admits several $C P$-violating parameters including the Cabibbo-Kobayashi-Maskawa phase, such as parameters in the Higgs potential, $C P$-violating Higgs VEVs, and additional Yukawa couplings with the fermions. How the induced fractional charge depends on the parameters is nontrivial, but it is calculable in principle. The effects could be useful to probe the $C P$ violation in the 2HDM using the Nambu monopole or dyon.

In this paper, we have considered a single Nambu monopole or dyon configuration with the two strings. In

\footnotetext{
${ }^{17}$ The Witten effect for the Nambu monopole in the SM was argued in Ref. [15].
}

practice, there can also be an antimonopole or antidyon on the string. Since the electric charges of the dyon and the antidyon are arbitrary, we can assign electric charges of the same sign for the pair, leading to an electrically repulsive force between them. This can change the fate of the dyons and monopoles in the early Universe as follows. In a realistic $2 \mathrm{HDM}$, the $U(1)_{a}$ symmetry should be explicitly broken to give a mass for the $\mathrm{CP}$-odd Higgs boson, which results in domain walls (or membranes) attached to the strings [45]. The strings pulled by the walls' tension collide with each other and reconnect, forming small string loops stretched by walls inside the loop. Such small loops immediately shrink and annihilate. However, if a string loop has a dyon pair with electric charges of the same sign, the dyons experience an electrically repulsive force, preventing the loop from shrinking at a certain loop size. Thus, the string loop with the dyon pair behaves as a long-lived particle and can be abundant in the present Universe. A quantitative discussion requires further studies, which we leave for future work.

\section{ACKNOWLEDGMENTS}

We would like to thank Masafumi Kurachi for discussion in the early stage of this work. We also thank Yoshihiko Abe, Ryuichiro Kitano, Masatoshi Yamada, and Wen Yin for useful discussions. The work is supported in part by Japan Society for the Promotion of Science Grant-in-Aid for Scientific Research [KAKENHI Grant No. No. JP19K03839 (M.E.), No. JP18J22733 (Y.H.), No. JP18H01217 (M.N.)], and also by Ministry of Education, Culture, Sports, Science and Technology KAKENHI Grant-in-Aid for Scientific Research on Innovative Areas, Discrete Geometric Analysis for Materials Design, No. JP17H06462 (M.E.) from the MEXT of Japan.

\section{APPENDIX A: FIERZ IDENTITIES}

In this Appendix we summarize the Fierz identities. For $S U(N)$ generators $T^{a}$ such that

$$
\operatorname{Tr}\left(T^{a} T^{b}\right)=\frac{1}{2} \delta^{a b}
$$

the following relations hold:

$$
\begin{array}{r}
\operatorname{Tr}\left(T^{a} X\right) \operatorname{Tr}\left(T^{a} Y\right)=\frac{1}{2} \operatorname{Tr}(X Y)-\frac{1}{2 N} \operatorname{Tr}(X) \operatorname{Tr}(Y), \\
\operatorname{Tr}\left(T^{a} X T^{a} Y\right)=\frac{1}{2} \operatorname{Tr}(X) \operatorname{Tr}(Y)-\frac{1}{2 N} \operatorname{Tr}(X Y),
\end{array}
$$

with arbitrary $N \times N$ matrices $X, Y$. 
Using these relations, we have

$$
\begin{aligned}
\left(\Phi^{\dagger} \sigma^{a} \Phi\right)\left(\Phi^{\dagger} \sigma^{a} \Phi\right) & =\operatorname{Tr}\left[\sigma^{a} \Phi \Phi^{\dagger} \sigma^{a} \Phi \Phi^{\dagger}\right] \\
& =2 \operatorname{Tr}\left(\Phi \Phi^{\dagger}\right) \operatorname{Tr}\left(\Phi \Phi^{\dagger}\right)-\operatorname{Tr}\left(\Phi \Phi^{\dagger} \Phi \Phi^{\dagger}\right) \\
& =\left(\Phi^{\dagger} \Phi\right)^{2} .
\end{aligned}
$$

Other useful relations can also be derived (see Ref. [12]).

\section{APPENDIX B: BACKGROUND GAUGE CONDITION}

Here we consider perturbations around a background field configuration in the $S U(2)_{W} \times U(1)_{Y}$ gauge theory. Due to the gauge symmetry, perturbations in the direction of the gauge orbit should be identified and any physical perturbations should be orthonormal to the gauge orbit. This is called the background gauge condition. Let $\delta W_{\mu}$, $\delta Y_{\mu}$ be the perturbations around the background fields $\bar{W}_{\mu}$, $\bar{Y}_{\mu}$. The orthonormality requires that the following conditions hold:

$$
\begin{gathered}
\left\langle\delta W_{\mu} \mid \delta_{\eta} W_{\mu}\right\rangle=0, \\
\left\langle\delta Y_{\mu} \mid \delta_{\chi} Y_{\mu}\right\rangle=0,
\end{gathered}
$$

where $\delta_{\eta} W_{\mu}$ and $\delta_{\chi} Y_{\mu}$ are the gauge transformations with the gauge functions $\eta^{a}(x)$ and $\chi(x)$, respectively.

Using $\delta_{\eta} W_{\mu}=\bar{D}_{\mu} \eta$ and $\delta_{\eta} Y_{\mu}=\partial_{\mu} \chi$, these conditions can be rewritten as

$$
\begin{gathered}
\int d^{4} x \operatorname{Tr}\left(\delta W_{\mu} \bar{D}^{\mu} \eta\right)=0, \\
\int d^{4} x \delta Y_{\mu} \partial^{\mu} \chi=0
\end{gathered}
$$

for any $\eta(x)$ and $\chi(x)$. By integrating them by parts, we obtain

$$
\bar{D}^{\mu} \delta W_{\mu}=\partial^{\mu} \delta Y_{\mu}=0,
$$

which are called the background gauge conditions.

\section{APPENDIX C: SINGULAR GAUGE FOR THE NAMBU MONOPOLE IN THE 2HDM}

Here we derive the Dirac quantization condition for the Nambu monopole in the 2HDM in a singular gauge. We again start from Eqs. (5.10)-(5.12). Recall that the vector $n^{a}$ is Eq. (5.25).

By performing the gauge transformation

$$
\begin{aligned}
U & =e^{i \sigma^{3} \varphi / 2} e^{i \sigma^{2} \Theta / 2} e^{-i \sigma^{3} \varphi / 2} \\
& =\left(\begin{array}{cc}
\cos \frac{\Theta}{2} & -\sin \frac{\Theta}{2} e^{-i \varphi} \\
\sin \frac{\Theta}{2} e^{i \varphi} & \cos \frac{\Theta}{2}
\end{array}\right),
\end{aligned}
$$

which is singular only at $\theta=\pi, n^{a}$ transforms as

$$
n \rightarrow U^{\dagger} n U=\frac{\sigma^{3}}{2},
$$

or, equivalently, $n^{a} \rightarrow(0,0,1)$. Under this transformation, the two doublets transform as

$$
\begin{gathered}
\Phi_{1} \rightarrow U^{\dagger} \Phi_{1}=\left(\begin{array}{c}
e^{-i \varphi}\left(f_{1} c_{1 / 2} \cos \frac{\Theta}{2}+h_{1} s_{1 / 2} \sin \frac{\Theta}{2}\right) \\
h_{1} s_{1 / 2} \cos \frac{\Theta}{2}-f_{1} c_{1 / 2} \sin \frac{\Theta}{2}
\end{array}\right), \\
\Phi_{2} \rightarrow U^{\dagger} \Phi_{2}=\left(\begin{array}{c}
\left(h_{2} c_{1 / 2} \cos \frac{\Theta}{2}+f_{2} s_{1 / 2} \sin \frac{\Theta}{2}\right) \\
e^{i \varphi}\left(f_{2} s_{1 / 2} \cos \frac{\Theta}{2}-h_{2} c_{1 / 2} \sin \frac{\Theta}{2}\right)
\end{array}\right),
\end{gathered}
$$

with $s_{1 / 2}=\sin \frac{\theta}{2}$ and $c_{1 / 2}=\cos \frac{\theta}{2}$. Equation (C3) is singular only at $\theta=\pi$, while Eq. (C4) is regular everywhere.

This singular transformation also gives a singularity at $\theta=\pi$ to the $S U(2)_{W}$ gauge field as

$$
\begin{aligned}
g W_{i} \rightarrow & U^{\dagger}\left(g W_{i}-i \partial_{i}\right) U \\
= & -\cos ^{2} \theta_{W} \frac{\sigma_{3}}{2} j(\theta) \cos \theta \partial_{i} \varphi+U^{\dagger}\left(\frac{\sigma_{a}}{2} \epsilon^{i a b} \frac{x^{b}}{r^{2}}-i \partial_{i}\right) U \\
= & -\cos ^{2} \theta_{W} \frac{\sigma_{3}}{2} j(\theta) \cos \theta \partial_{i} \varphi+\frac{\sigma_{3}}{2} \frac{1-\cos \theta}{r \sin \theta} \hat{\varphi}_{i} \\
& +\mathcal{O}(\theta-\pi),
\end{aligned}
$$

where the last term denotes some regular terms that vanish at $\theta \rightarrow \pi$. Equation (C5) has a singularity at $\theta=\pi$. In this gauge, the electromagnetic gauge field is [note that $Y_{i} \rightarrow 0$ at $\theta \rightarrow \pi$ due to $k(\pi)=0$ ]

$$
\begin{aligned}
A_{i} & =-\sin \theta_{W} W_{i}^{3}+\cos \theta_{W} Y_{i} \\
& =\frac{\sin \theta_{W}}{g}(1-\cos \theta) \partial_{i} \varphi+\mathcal{O}(\theta-\pi),
\end{aligned}
$$

and we have

$$
\partial_{[i} A_{j]}=\frac{2 \pi \sin \theta_{W}}{g}\left(1-\frac{z}{|z|}\right) \delta(x) \delta(y) .
$$

On the other hand, the $Z$ gauge field is

$$
\begin{aligned}
Z_{i} & =-\cos \theta_{W} W_{i}^{3}-\sin \theta_{W} Y_{i} \\
& =\frac{\cos \theta_{W}}{g r} \frac{1-\cos \theta}{\sin \theta} \hat{\varphi}_{i}+\mathcal{O}(\theta-\pi) \\
& =\frac{\cos \theta_{W}}{g}(1-\cos \theta) \partial_{i} \varphi+\mathcal{O}(\theta-\pi),
\end{aligned}
$$

which also has a line singularity at $\theta=\pi$.

Let us consider a test particle moving around the line singularity, i.e., with $\varphi$ from 0 to $2 \pi$ and $\theta \sim \pi$, and calculate the $\mathrm{AB}$ phase $\theta_{A B}$ :

$$
\exp \left[i \theta_{A B}\right]=\exp \left[i \oint_{\theta \approx \pi} d x_{i}\left(e Q A_{i}+g_{Z} T_{Z} Z_{i}\right)\right],
$$

where $Q$ and $T_{Z}$ are defined in Eqs. (5.47) and (5.48). 
Equation (C9) reads

$$
\begin{aligned}
(\mathrm{C} 9) & =\exp \left[i \oint_{\theta \approx \pi} d x_{i}\left(-g T_{3} W_{i}^{3}+g^{\prime} \frac{Y}{2} Y_{i}\right)\right] \\
& =\exp \left[-i T_{3} \oint_{\theta \approx \pi} d x_{i}(1-\cos \theta) \partial_{i} \varphi\right] \\
& =\exp \left(-4 \pi i T_{3}\right) .
\end{aligned}
$$

Since the line singularity is a gauge artifact, it must not be observed, and we thus obtain the condition

$$
4 \pi T_{3}=2 \pi n(n \in \mathbb{Z}) \Leftrightarrow T_{3}=\frac{n}{2},
$$

which is the same condition as Eq. (5.52) derived from the argument on the fiber bundle. Note that we have to take into account the line singularities in not only $A_{i}$ but also $Z_{i}$.
[1] P. A. M. Dirac, Proc. R. Soc. A 133, 60 (1931).

[2] T. T. Wu and C. N. Yang, Phys. Rev. D 12, 3845 (1975).

[3] G. 't Hooft, Nucl. Phys. B79, 276 (1974).

[4] A. M. Polyakov, JETP Lett. 20, 194 (1974).

[5] H. Georgi and S. Glashow, Phys. Rev. Lett. 32, 438 (1974).

[6] B. Julia and A. Zee, Phys. Rev. D 11, 2227 (1975).

[7] Y. Nambu, Phys. Rev. D 10, 4262 (1974).

[8] N. Seiberg and E. Witten, Nucl. Phys. B431, 484 (1994).

[9] N. Seiberg and E. Witten, Nucl. Phys. B426, 19 (1994); B430, 485(E) (1994).

[10] C. Castelnovo, R. Moessner, and S. L. Sondhi, Nature (London) 451, 42 (2008).

[11] M. W. Ray, E. Ruokokoski, S. Kandel, M. Mttnen, and D. S. Hall, Nature (London) 505, 657 (2014).

[12] Y. Nambu, Nucl. Phys. B130, 505 (1977).

[13] T. Vachaspati, Phys. Rev. Lett. 87, 251302 (2001).

[14] R. Poltis and D. Stojkovic, Phys. Rev. Lett. 105, 161301 (2010).

[15] T. Vachaspati, Nucl. Phys. B439, 79 (1995).

[16] T. Vachaspati, Phys. Rev. Lett. 68, 1977 (1992); 69, 216(E) (1992).

[17] T. Vachaspati, Nucl. Phys. B397, 648 (1993).

[18] A. Achucarro and T. Vachaspati, Phys. Rep. 327, 347 (2000); 327, 347 (2000).

[19] R. H. Brandenberger and A.-C. Davis, Phys. Lett. B 308, 79 (1993).

[20] M. Barriola, Phys. Rev. D 51, R300 (1995).

[21] M. Eto, K. Konishi, M. Nitta, and Y. Ookouchi, Phys. Rev. D 87, 045006 (2013).

[22] M. James, L. Perivolaropoulos, and T. Vachaspati, Phys. Rev. D 46, R5232 (1992).

[23] M. James, L. Perivolaropoulos, and T. Vachaspati, Nucl. Phys. B395, 534 (1993).

[24] J. F. Gunion, H. E. Haber, G. L. Kane, and S. Dawson, Front. Phys. 80, 1 (2000).

[25] G. C. Branco, P. M. Ferreira, L. Lavoura, M. N. Rebelo, M. Sher, and J. P. Silva, Phys. Rep. 516, 1 (2012).

[26] H. P. Nilles, Phys. Rep. 110, 1 (1984).

[27] H. E. Haber and G. L. Kane, Phys. Rep. 117, 75 (1985).

[28] M. Trodden, Rev. Mod. Phys. 71, 1463 (1999).

[29] S. Kanemura, M. Kikuchi, and K. Yagyu, Nucl. Phys. B896, 80 (2015).

[30] S. Kanemura, K. Tsumura, K. Yagyu, and H. Yokoya, Phys. Rev. D 90, 075001 (2014).
[31] F. Kling, J. M. No, and S. Su, J. High Energy Phys. 09 (2016) 093.

[32] J. Haller, A. Hoecker, R. Kogler, K. Mönig, T. Peiffer, and J. Stelzer, Eur. Phys. J. C 78, 675 (2018).

[33] H. La, arXiv:hep-ph/9302220.

[34] M. A. Earnshaw and M. James, Phys. Rev. D 48, 5818 (1993).

[35] L. Perivolaropoulos, Phys. Lett. B 316, 528 (1993).

[36] G. Bimonte and G. Lozano, Phys. Lett. B 326, 270 (1994).

[37] I. P. Ivanov, Phys. Rev. D 77, 015017 (2008).

[38] Y. Brihaye, J. Phys. A 41, 405401 (2008).

[39] J. Grant and M. Hindmarsh, Phys. Rev. D 64, 016002 (2001).

[40] J. Grant and M. Hindmarsh, Phys. Rev. D 59, 116014 (1999).

[41] C. Bachas, P. Tinyakov, and T. N. Tomaras, Phys. Lett. B 385, 237 (1996).

[42] R. A. Battye, G. D. Brawn, and A. Pilaftsis, J. High Energy Phys. 08 (2011) 020.

[43] G. D. Brawn, Ph.D. thesis, University of Manchester, 2011.

[44] M. Eto, M. Kurachi, and M. Nitta, J. High Energy Phys. 08 (2018) 195.

[45] M. Eto, M. Kurachi, and M. Nitta, Phys. Lett. B 785, 447 (2018).

[46] N. Chen, T. Li, Z. Teng, and Y. Wu, J. High Energy Phys. 10 (2020) 081.

[47] R. A. Battye, A. Pilaftsis, and D. G. Viatic, arXiv: 2006.13273.

[48] C. Bachas and T. N. Tomaras, Phys. Rev. Lett. 76, 356 (1996).

[49] A. Riotto and O. Tornkvist, Phys. Rev. D 56, 3917 (1997).

[50] G. R. Dvali and G. Senjanovic, Phys. Rev. Lett. 71, 2376 (1993).

[51] G. R. Dvali and G. Senjanovic, Phys. Lett. B 331, 63 (1994).

[52] C. Bachas, B. Rai, and T. N. Tomaras, Phys. Rev. Lett. 82, 2443 (1999).

[53] A. P. Balachandran, S. Digal, and T. Matsuura, Phys. Rev. D 73, 074009 (2006).

[54] E. Nakano, M. Nitta, and T. Matsuura, Phys. Rev. D 78, 045002 (2008).

[55] M. Eto and M. Nitta, Phys. Rev. D 80, 125007 (2009).

[56] M. Eto, M. Nitta, and N. Yamamoto, Phys. Rev. Lett. 104, 161601 (2010). 
[57] M. Eto, Y. Hirono, M. Nitta, and S. Yasui, Prog. Theor. Exp. Phys. 2014, 012D01 (2014).

[58] M. Eto, Y. Hamada, M. Kurachi, and M. Nitta, Phys. Lett. B 802, 135220 (2020).

[59] M. Eto, Y. Hamada, M. Kurachi, and M. Nitta, J. High Energy Phys. 07 (2020) 004.

[60] Y. M. Shnir, Magnetic Monopoles, Text and Monographs in Physics (Springer, Berlin, Heidelberg, 2005).

[61] H. B. Nielsen and P. Olesen, Nucl. Phys. B61, 45 (1973).
[62] A. A. Abrikosov, Zh. Eksp. Teor. Fiz. 32, 1442 (1957) [Sov. Phys. JETP 5, 1174 (1957)].

[63] M. Hindmarsh and M. James, Phys. Rev. D 49, 6109 (1994).

[64] O. Tornkvist, arXiv:hep-ph/9805255.

[65] B. Grzadkowski, M. Maniatis, and J. Wudka, J. High Energy Phys. 11 (2011) 030.

[66] E. Witten, Phys. Lett. B 86, 283 (1979).

[67] C. G. Callan, Jr., Phys. Rev. D 26, 2058 (1982). 